\title{
GLOBAL RESIDUES AND INTERSECTIONS ON A COMPLEX MANIFOLD
}

BY

JAMES R. KING(1)

\begin{abstract}
This paper is the study of a class of forms $\eta$ on a complex manifold $V$ which are smooth on $V-W$ and have poles of kernel type on a complex submanifold $W$ of codimension $d$; such a form is one whose pull-back to the monoidal transform of $V$ along $W$ has a logarithmic pole. A global existence theorem is proved which asserts that any smooth form $\varphi$ on $W$ of filtration $s$ (no $(p, q)$ components with $p<s$ ) is the residue of a form $\eta$ of filtration $s+d$ such that $d \eta$ is smooth on $V$. This result is used to construct global kernels for $\bar{\partial}$ which establish similar global existence theorems for $W$ with singularities. We then establish formulas connecting intersection and wedge product on the d-cohomology theory of Dolbeault which preserve the Hodge filtration. A number of results are also proved on the integrability of $f^{*} \eta$ where $f$ is a rather general holomorphic map.
\end{abstract}

Introduction. This paper contains the study of a certain class of residue forms on a complex manifold and the application of these forms to establish some imtersection formulas. The formula of $\$ 7$ in particular, which has applications to algebraic geometry, depends strongly on the complex structure of the manifold. Therefore, the residue theory of this paper is based on the $d^{\prime \prime}$ operator and Dolbeault cohomology rather than the $d$ operator and de Rham cohomology.

We will use the theory of currents, or forms with coefficients that are Schwartz distributions. In general on a manifold we may write such a current $v$ as $d u+[\varphi]$ where $u$ is a current and $[\varphi]$ is the current defined by a smooth form. If $u$ is the current defined by a locally $L^{1}$ form $\eta$, we say that this is a residue formula for $v$ and that $\eta$ is a residue form. Our interest will center on the case when $v$ is given by integration over a submanifold or subvariety and $\eta$ is $C^{\infty}$ except for poles of a certain type along the support of $v$.

The connection of residue formulas with intersections is in trying to define $v \wedge u$ for two currents, which cannot be done in general. If $v=d[\eta]+[\varphi]$ and $u$ is of order zero, i.e., has coefficients which are measures and if $\eta$ is integrable with respect to $u$, then we can set $v \wedge u=d(\eta \wedge u)-\varphi \wedge u \pm \eta \wedge d u$, if $\eta \wedge d u$ is defined also.

In [28] de Rham shows that if $u$ and $v$ are the currents of integration over two transversal smooth chains then $u \wedge v$ is defined and equals the current of integration over the intersection. He uses the method of smoothing operators;

Received by the editors November 3, 1972.

AMS (MOS) subject classifications (1970). Primary 32A25, 32C30; Secondary 32C35, 32G20.

Key words and phrases. Residue forms, intersection of analytic sets, Dolbeault cohomology, kernels on complex manifolds.

(1) Supported in part by National Science Foundation grant GP-7952X3.

Copyright $\odot$ 1974, American Mathematical Society 
this method will not work for some of the intersection formulas studied here because smoothing does not preserve the $(p, q)$ type of a current. In the residue approach above, however, if $v, \eta$ and $\varphi$ have certain types, then so will the exterior product $v \wedge u$.

In $\$ \S 1-3$ we define forms with simple kernel type along a complex submanifold $W$ and establish the existence of global residue forms $\eta$ of this type so that $[W]=d[\eta]+[\psi]$. This is done by showing that the subcomplex of the complex of all currents generated by these forms gives Dolbeault and de Rham resolutions of $\mathbf{C}, \boldsymbol{\Omega}^{p}$, and $\hat{\Omega}^{p}$.

In $\$ 4$ we use this existence theory to construct global kernels for the $d^{\prime \prime}$ operator on $V \times V$; the operator associated to such a residue kernel gives global residue formulas for more general currents, including integration currents over complex subvarieties.

These residue forms have remarkable integration properties with respect to pull-back by holomorphic maps; for example, under quite weak conditions the restriction of such a form to a subvariety is locally $L$ on the subvariety. These results are proved in $\$ 5$, along with a generalization to the continuity theorem of [19] to include residue forms.

In $\$ 6$, using these residue forms we define intersection of complex analytic subvarieties as $v \wedge u$ by the process indicated above. This definition coincides with the usual one.

An intersection formula for chains whose boundaries are analytic subvarieties is proved in \$7; this can be applied to the theory of intermediate Jacobian varieties developed in [10]. In this section a theory of intersection of currents with a complex submanifold is developed.

The operation of taking a residue defines an operation in cohomology and a dual operation in homology. We discuss how this relates to our theory in $\S 8$.

The methods of proof are mostly quite elementary except for the use of Hironaka's resolution of singularities to simplify certain arguments about integrability in $\$ \S 5$ and 6. This approach avoids the direct use of Federer's geometric measure theory found in [18] and [19], but ideas from this theory are still found in this paper. The key idea in all the paper is the use of the strong functorial properties of the logarithmic differential $t^{-1} d t$.

We have not discussed the real case here, but much of the intersection theory would carry over.

Finally, we would like to mention some other recent work in this area; a general survey can be found in [24]. In [13] and [14] is an approach to residues using poles of higher order than in this paper. Also, Poly has announced results about residues for the $d$-operator which are closer to those of this paper [27]. He establishes global residue formulas and applies them to study the homology of subvarieties and the cohomology of their complements. For this reason, although our methods here would also yield some results in this direction, we have only mentioned them cursorily in $\$ 8$. 
0. Preliminaries. If $V$ is an oriented (always paracompact) $C^{\infty}$ manifold, we denote by $A^{r}(V)$ the space of $\mathbf{C}$-valued $r$-forms and by $A_{c}^{r}(V)$ the subspace of forms with compact support. The dual spaces of $A_{c}^{r}(V)$ and $A^{r}(V)$ in their usual $C^{\infty}$ topologies [29] are, respectively, $\mathscr{D}_{r}^{\prime}(V)$, the space of currents of dimension $r$, and $\mathcal{E}_{r}^{\prime}(V) \subset \mathscr{D}_{r}^{\prime}(V)$, the space of currents with compact support. If $\operatorname{dim}_{R} V=n$, it will sometimes be convenient to write $\mathscr{D}^{\prime n-r}(V)=\mathscr{D}_{r}^{\prime}(V)$ and $\mathcal{E}^{\prime n-r}(V)$ $=\mathcal{E}_{r}^{\prime}(V)$.

There is a natural injection $A^{r}(V) \hookrightarrow \Phi^{\prime \prime}(V)$ (cf. (1.1)) and the exterior derivative may be extensed to $d: \mathscr{Q}^{\prime r}(V) \rightarrow \mathscr{Q}^{\prime r+1}(V)$ by setting

$$
d u(\varphi)=(-1)^{r+1} u(d \varphi)
$$

for some $u \in \mathscr{Q}^{\prime r}(V)$ and any $\varphi \in A_{c}^{n-r-1}(V)$.

If $V$ is a complex manifold of complex dimension $m$, we also have the spaces $A^{p, q}(V)$ and $A_{c}^{p, q}(V)$ of $C^{\infty}(p, q)$ forms and $C^{\infty}(p, q)$ forms with compact support. There are also $\mathscr{D}^{\prime p, q}(V)$ and $\mathcal{E}^{\prime p, q}(V)$, where $\mathscr{D}^{\prime p, q}(V) \subset \mathscr{D}^{\prime p+q}(V)$ is the dual space of $A_{c}^{m-p, m-q}(V)$, etc. The operator $d=d^{\prime}+d^{\prime \prime}$ as usual, where $d^{\prime \prime}: 囚^{\prime p, q}(V) \rightarrow \Phi^{\prime p, q+1}(V)$ is given by

$$
d^{\prime \prime} u(\varphi)=(-1)^{p+q+1} u\left(d^{\prime \prime} \varphi\right)
$$

for any $\varphi \in A_{c}^{m-p, m-q-1}(V)$. The currents in $\mathscr{D}^{p, q}(V)$ can be considered as $(p, q)$ forms with distribution coefficients.

If $f: V_{1} \rightarrow V_{2}$ is a $C^{\infty}$ map, it induces $f_{*}: \mathcal{E}_{r}^{\prime}\left(V_{1}\right) \rightarrow \mathcal{E}_{r}^{\prime}\left(V_{2}\right)$ by

$$
f_{*} u(\varphi)=u\left(f^{*} \varphi\right)
$$

for any $\varphi \in A^{r}\left(V_{2}\right)$, where $f^{*}: A^{r}\left(V_{2}\right) \rightarrow A^{r}\left(V_{1}\right)$ is the usual pull-back map. If the $V_{i}$ are complex manifolds and $f$ is holomorphic, then $f_{*}: \mathcal{E}^{\prime p, q}\left(V_{1}\right) \rightarrow \mathcal{E}^{\prime p-d, q-d}\left(V_{2}\right)$, where $d=\operatorname{dim}_{\mathrm{C}} V_{1}-\operatorname{dim}_{\mathrm{C}} V_{2}$. The map $f_{*}$ can be extended to all currents $u$ such that $f \mid$ supp $u$ is proper; if $f$ is proper (i.e., $f^{-1}$ of any compact set is compact) then $f_{*}$ extends to all of $\mathscr{D}_{r}^{\prime}\left(V_{1}\right)$.

The sheaves $\mathfrak{A}_{V}^{r}, \mathfrak{A}^{p}, q, \mathfrak{K}_{V}^{r}$, and $\mathfrak{K}_{\mathcal{P}} \boldsymbol{P}^{q}$ are the sheaves of germs of forms or currents in $A^{r}(V), A^{p, q}(V), \mathscr{D}^{\prime r}(V)$, and $\mathscr{D}^{\prime p, q}(V)$, respectively. For complex $V$, the sheaves $\theta_{V}$ and $\Omega_{V}^{p}$ are the sheaves of holomorphic functions and holomorphic $(p, 0)$ forms. The sheaf $\hat{\Omega}_{V}^{p} \subset \Omega_{V}^{p}$ is the sheaf of holomorphic forms $\psi$ with $d \psi=0 ; \hat{\Omega}_{V}^{0}=\mathrm{C}_{V}$ the constant sheaf.

In general, given a sheaf $\mathscr{F}$ on $V$, we will write $\Gamma \mathscr{F}$ for $\Gamma(V, \mathscr{F})$. Also if

$$
C^{0} \stackrel{8}{\rightarrow} C^{1} \stackrel{8}{\rightarrow} C^{2} \rightarrow \cdots
$$

is a cochain complex, $H^{p}\left(C^{\cdot}, \delta\right)$ or just $H^{p}\left(C^{\cdot}\right)$ is the $p$ th cohomology group of the complex.

For the theory of currents, see [28] or [29], for manifolds and sheaves [16].

A complex space $X$ is a ringed space locally isomorphic to a complex analytic subvariety in $\mathbf{C}^{N}$ with the holomorphic functions on the subvariety. All complex 
spaces will be reduced (see [12]). We denote by $\operatorname{Reg} X$, Sing $X$, the locus of regular and singular points of $X$.

As in [2], $A^{p, q}(X)$ may be defined locally for $X \subset C^{N}$ by $A^{p, q}(X)$ $=A^{p, q}\left(C^{N}\right) / I_{X}^{p, q}$, where $I_{X}^{p, q}$ is the space of $(p, q)$ forms $\varphi$ such that $i^{*} \varphi=0$, where $i$ : $\operatorname{Reg} X \rightarrow C^{N}$ is the inclusion. The currents $\mathscr{D}^{\prime p, q}(X) \subset \mathscr{D}^{\prime p, q}\left(C^{N}\right)$ are those currents that annihilate $I_{X}^{N-p, N-q}$. We also define the other $A, \mathscr{D}$, and $\mathcal{E}$ spaces as for $V ; f^{*}$ and $f_{*}$ are defined (this requires an argument).

Given a collection $G^{p, q}$ indexed by integers $p$ and $q$ we write

$$
F^{p} G^{r}=\sum_{k \geq p} G^{k, r-k}
$$

Thus $F^{p} A^{r}(V), F^{p} \mathcal{K}_{V}^{r}$, etc., are forms and currents $\sum a_{I J} d z^{I} d z^{-J}$ with $|I| \geq p$.

Given a complex manifold or space, dimension means complex dimension unless otherwise specified. For $W \subset V$, codimension $W=\operatorname{dim} V-\operatorname{dim} W$.

A resolution of the singularities of a complex space $X$ of pure dimension $n$ is a proper holomorphic map $\tau=\hat{X} \rightarrow X$ of a complex $n$-manifold $\hat{X}$ such that there is a subvariety $S \subset X$ of dimension $<n$ such that $\tau$ maps $X-\tau^{-1}(S)$ biholomorphically onto $X-S$. Given any subvariety $Y \subset X$ with $\operatorname{dim} Y<n$, there exists such a $\tau$ such that $\tau^{-1}(Y)$ is a divisor with normal crossings; but we shall only need the local version of this theorem found in Hironaka's original paper [15]. A divisor with normal crossings is a subvariety of codimension one such that in some $\left(z_{1}, \ldots, z_{n}\right)$ coordinates $Y=\left\{z_{1} \cdots z_{k}=0\right\}$ for some $k$, i.e., $Y$ is a union of coordinate hyperplanes.

1. Residue formulas. If $\varphi$ is a differential $r$-form with locally (Lebesgue) summable coefficients on a smooth oriented $n$-manifold $V$, then $\varphi$ defines a current $[\varphi] \in \mathscr{Q}^{\prime \prime}(V)$, a linear functional on $A_{c}^{n-r}(V)$. Take any $\psi \in A_{c}^{n-r}(V)$ and set

$$
[\varphi](\psi)=\int_{V} \varphi \wedge \psi
$$

This integral is defined since $\varphi \wedge \psi$ is a locally summable form with compact support and hence summable.

One can also define another current $d[\varphi] \in \mathscr{Q}^{\prime r+1}(V)$ by formula $(0.1)$. If $\varphi$ happened to be a continuously differentiable form-a form in $A^{r}(V)$ for example-then Stokes's theorem implies $d[\varphi]=[d \varphi]$, i.e., $\int \varphi \wedge d \psi$ $=(-1)^{r+1} \int d \varphi \wedge \psi$ for all $\psi \in A_{c}^{n-r-1}(V)$.

If $\varphi$ is smooth in the complement of a closed set of measure zero, the form $d \varphi$ is defined almost everywhere, and if $d \varphi$ is locally summable the current [ $d \varphi$ ] is defined. In this case it may be true that $d[\varphi] \neq[d \varphi]$; an expression for the difference between the two sides is a kind of residue formula.

This last statement can best be explained by several illustrative examples. After this we will turn to the question of writing an interesting current, such as integration over a submanifold, in the form $d[\varphi]-[d \varphi]$. 
Example 1.2. Let $f$ be a holomorphic function on a complex manifold $V$. If $W$ is the analytic subset defined by $\{f=0\}$, then

$$
d\left[\frac{1}{2 \pi i} \frac{d f}{f}\right](\psi)=\int_{W} n_{f} \psi,
$$

where $n_{f}(x)$ is the order of vanishing of $f$ at $x \in W$. This was first proved by Lelong; for his proof see [22, p. 224]. It is also shown in the proof of Lemma 6.1.

We will prove the special case where $w=C$ and $f=z$ just to illustrate the idea. Let $\psi$ be a smooth function with compact support on $\mathbf{C}$; then by definition

$$
\begin{aligned}
d\left[\frac{1}{2 \pi i} \frac{d z}{z}\right](\psi) & =(2 \pi i)^{-1} \int_{\mathbf{c}} \frac{d z}{z} \wedge d \psi=\lim _{\varepsilon \rightarrow 0}(2 \pi i)^{-1} \int_{|z| \geq e} \frac{d z}{z} \wedge d \psi \\
& =\lim _{\varepsilon \rightarrow 0}(2 \pi i)^{-1} \int_{|z|-e} \psi \frac{d z}{z}=\psi(0) \lim _{e \rightarrow 0}(2 \pi i)^{-1} \int_{|z|-e} \frac{d z}{z}=\psi(0)
\end{aligned}
$$

by Stokes's theorem.

The crucial property of $(2 \pi i z)^{-1} d z$ is that the integral on the circle $|z|=\varepsilon$ tends to (in this case equals) one. In general the integral of $(2 \pi i f)^{-1} d f$ around a normal circle at $x \in W$ is $n_{f}(x)$, although the singularities of $W$ create technical difficulties.

To represent integration over sets $W$ of higher codimension it suffices to find a form of higher degree whose integral over normal $\varepsilon$-spheres tends to one.

Example 1.4. Let $f_{1}, \ldots, f_{r}$ be holomorphic functions on a complex manifold $V$ and suppose $W=\left\{f_{1}=\cdots=f_{r}=0\right\}$ is a complex subvariety of pure complex codimension $k$. Then if we set $\eta=(1 / 2 \pi i) d^{\prime} \log \left(\left|f_{1}\right|^{2}+\cdots+\left|f_{r}\right|^{2}\right)$ and $\omega=d \eta$,

$$
\int_{W} n_{F} \psi=d\left[\eta \wedge \omega^{k-1}\right](\psi)-\left[\omega^{k}\right](\psi),
$$

where $n_{F}(x)$ is the multiplicity of $\left(f_{1}, \ldots, f_{r}\right)$ at $x \in W$. A proof is in [18], and similar results will be proved in $\$ 6$.

In particular if $r=k, \omega^{k}=0$; if $r=1$, we are reduced to the previous example. The simplest case in higher codimension is $V=\mathbf{C}^{n}, \quad \eta$ $=\left(2 \pi i^{-1}\right) d^{\prime} \log \left(\left|z_{1}\right|^{2}+\cdots+\left|z_{n}\right|^{2}\right)$. Here the integral of the form $\eta \wedge \omega^{n-1}$ over an $\varepsilon$-sphere around the origin is 1 ; and $d\left[\eta \wedge \omega^{n-1}\right](\psi)=\psi(0)$. This singular kernel is the Bochner-Martinelli kernel.

Whenever $W$ is a complex $k$-dimensional subvariety of a complex manifold $V$ of dimension $m$, if we are given a multiplicity function $n_{W}(x)$ for $x \in W$ which assigns a constant (usually integer) multiplicity to each irreducible component of $W$, we can define a current $[W] \in \Phi^{\prime m-k, m-k}(V)$ by

$$
[W](\psi)=\int_{\operatorname{Reg} W} n_{W} \psi
$$


for any $\psi \in A_{c}^{2 k}(V)$. The integral over the regular points of $W$ is well defined and $d[W]=0[31]$ or $[19$, p. 200].

Thus the examples above can be written as an equation of currents:

$$
[W]=d\left[\eta \wedge \omega^{m-k-1}\right]-\left[\omega^{m-k}\right]
$$

where $\omega^{m-k} \equiv 0$ if $r=m-k$.

Generally there will not be global holomorphic functions defining a given subvariety $W$. The next example treats the simplest case of this phenomenon.

Example 1.8. Let $W$ be a complex subvariety of codimension one in the complex manifold $V$; then there is a line bundle $L$ and a holomorphic section $\sigma$ of $L$ such that the divisor $\{\sigma=0\}=W$. We put a hermitian metric on $L$. Locally the square of the length $|\sigma|^{2}=g_{\alpha}\left|\sigma_{\alpha}\right|^{2}$, where $\sigma_{\alpha}$ is the local expression of $\sigma$.

Then as a corollary of Example 1.2

$$
\int_{W} \psi=d[\eta](\psi)-[d \eta](\psi)
$$

where

$$
\eta=(2 \pi i)^{-1} d^{\prime} \log |\sigma|^{2}=(2 \pi i)^{-1}\left(d^{\prime} \log g_{\alpha}+\sigma_{\alpha}^{-1} d \sigma_{\alpha}\right) .
$$

But $d \eta=(2 \pi i)^{-1} d^{\prime \prime} d^{\prime} \log g_{\alpha}=-c(L)$, the Chern class of $L$, a smooth form.

Thus we have, writing $[W]$ for integration over $W$, the equation of currents:

$$
[W]=d[\eta]+[c(L)]
$$

2. Global residue forms. The last example above illustrates two important points. First, although in the local Examples 1.2 and 1.4 one can write $[W]=d[\eta]$ for some $\eta$, there is a topological obstruction to doing this globally. Secondly, although the de Rham theorem for currents merely implies that one can always write $[W]=d u+\varphi$, where $\varphi$ is a $C^{\infty}$ form and $u$ is a current, we can hope to choose $u$ and $\varphi$ judiciously so as to reflect the geometry of the situation.

In this section, assuming $W$ is nonsingular, we define a class of locally integrable forms which is large enough that we can write $u=[\eta]$ for some $\eta$ in this class.

We will make extensive use of fine resolutions of sheaves to get global residue formulas.

Proposition 2.1. If

$$
0 \rightarrow \mathfrak{K} \rightarrow \mathscr{F}^{0} \stackrel{\delta}{\rightarrow} \mathscr{F}^{1} \stackrel{\delta}{\rightarrow} \ldots \stackrel{\delta}{\rightarrow} \mathscr{F}^{n} \stackrel{\delta}{\rightarrow} \ldots
$$

is a fine resolution of the sheaf $\Re$ on $V$, then

$$
H^{p}(V, \mathscr{T}) \simeq H^{p}\left(\Gamma \mathscr{F}^{*}\right)=\frac{\operatorname{Ker}\left(\delta: \Gamma^{p} \rightarrow \Gamma^{p+1}\right)}{\operatorname{im}\left(\delta: \Gamma_{\mathscr{F}^{p-1}} \rightarrow \Gamma^{p}\right)}
$$


Proof. See [16].

Proposition 2.2. The sheaves $\mathfrak{A P}^{p, q}$ and $\mathfrak{K C}^{p, q}$ are fine sheaves; furthermore, any subsheaf of $\mathfrak{K}^{p-q}$ which is an $\mathfrak{X}_{0}^{0}$-module is fine.

Proof. Since such a sheaf admits multiplication by $C^{\infty}$ functions, it admits a partition of unity.

Corollary 2.3.

$$
\begin{aligned}
& H^{q}\left(V, \hat{\Omega}^{p}\right) \simeq H^{q}\left(\Gamma F^{p} \mathscr{X}^{p^{+}}, d\right) \simeq H^{q}\left(\Gamma F^{p} \mathcal{K}_{P^{+}}, d\right), \\
& H^{q}\left(V, \Omega^{p}\right) \simeq H^{q}\left(\Gamma \mathscr{A} P_{j}^{*}, d^{\prime \prime}\right) \simeq H^{q}\left(\Gamma \mathcal{K P}, d^{\prime \prime}\right) .
\end{aligned}
$$

These are the de Rham and Dolbeault theorems for forms and currents; notice that $H^{q}\left(V, \hat{\Omega}^{0}\right)=H^{q}(V, \mathrm{C})$ and $F^{0} \mathfrak{U}^{r}=\mathfrak{A}^{r}$, etc.

Proof. By Propositions 2.1 and 2.2 this follows from the following exact sequences of sheaves:

$$
\begin{aligned}
& \text { (i) } \quad 0 \rightarrow \hat{\Omega}^{p} \rightarrow F^{p} \mathfrak{X} \mathcal{P} \stackrel{d}{\rightarrow} F^{p} \mathfrak{A P}^{p+1} \stackrel{d}{\rightarrow} \cdots, \\
& \text { (ii) } \quad 0 \rightarrow \hat{\Omega}^{p} \rightarrow F^{p} K_{\ell}^{p} \rightarrow F^{p} \mathfrak{U P}^{p+1} \rightarrow \cdots \text {, } \\
& \text { (iii) } \quad 0 \rightarrow \Omega^{p} \rightarrow \mathfrak{A P}^{p^{0}} \stackrel{d^{*}}{\longrightarrow} \mathfrak{A P}^{p^{1}} \stackrel{d^{*}}{\longrightarrow} \cdots \text {, } \\
& \text { (iv) } \quad 0 \rightarrow \Omega^{p} \rightarrow \operatorname{KCP}^{0 .} \stackrel{d^{\mu}}{\longrightarrow} \operatorname{KCP}^{p .0} \stackrel{d^{\mu}}{\longrightarrow} \cdots \text {. }
\end{aligned}
$$

The exactness follows from the Poincare and Dolbeault lemmas for forms and currents and from the regularity of solutions of $d u=0, d^{\prime \prime} u=0$, for $u$ $\left.\in \mathscr{D}^{\prime p, 0}(V)=\Gamma \mathcal{K P}^{0}, 06\right]$.

Corollary 2.5. Let $W$ be a $k$-dimensional complex subvariety of a complex $n$ manifold $V$; then, if $r=n-k$

$$
\begin{aligned}
& {[W]=d u+[\varphi],} \\
& {[W]=d^{\prime \prime} u+[\psi],}
\end{aligned}
$$

where $u, v$ are currents in $F^{r} \mathscr{D}^{\prime 2 r-1}(V), \mathscr{D}^{\prime r,-1}(V)$ and $\varphi, \psi$ are smooth forms in $F^{r} A^{2 r}(V), A^{r, r}(V)$.

Proof. There is a natural injection of complexes $\Gamma \mathfrak{X}_{V}=A(V) \hookrightarrow \Gamma \varkappa_{V}^{*}$ $=\mathscr{D}^{\prime \prime}(V)$ given by (1.1). Since the cohomology is the same by Corollary 2.3 , each $d$ or $d^{\prime \prime}$ closed current is cohomologous to a smooth form.

The rest of this section will be devoted to defining forms of kernel type along $W, A^{r}(V\{W\})$, where $W$ is a complex submanifold of $V$. All the forms $\eta \wedge \omega^{r}$ and $\omega^{r}$ of the examples in $\$ 1$ will be included in this space by Proposition 2.18. Since all these forms will be locally summable, $A^{r}(V\{W\}) \hookrightarrow \Phi^{\prime r}(V)$.

In the interest of clarity, if not of logical progression, we will state here the rest of the important definitions and propositions of this section; the proofs, as noted 
will follow trivially from Definition 2.17 and the Dolbeault lemma, Proposition 3.1.

Definition 2.8. The spaces of currents of simple residue type along the submanifold $W$ are

$$
\begin{aligned}
\mathscr{D}^{\prime r}(V\{W\}) & =\left\{u \in \mathscr{D}^{\prime r}(V): u=\left[\eta_{0}\right]+d\left[\eta_{1}\right], \eta_{i} \in A^{r-i}(V\{W\})\right\}, \\
\mathscr{D}^{\prime p, q}(V\{W\}) & =\mathscr{D}^{\prime p, q}(V) \cap \mathscr{D}^{\prime p+q}(V\{W\}),
\end{aligned}
$$

and similarly for $\mathcal{E}^{\prime r}(V\{W\}), \mathcal{E}^{\prime p, q}(V\{W\})$.

The space $\sum A^{r}(V\{W\})$ is closed under multiplication by forms in $A^{s}(V)$. This is also true of $\sum \mathscr{D}^{\prime \prime}(V\{W\})$ since $\psi \wedge d[\eta]= \pm d[\psi \wedge \eta] \mp[d \psi \wedge \eta]$. In particular, a current $u$ in $\mathscr{D}^{\prime r}(V\{W\})$ can be broken up by a partition of unity $\sum \lambda_{i}$ and each $\lambda_{i} u \in \mathscr{D}^{\prime}(V\{W\})$ also.

Proposition 2.9. If $\varphi \in A^{p, q}(W), i_{*}[\varphi] \in \mathscr{Q}^{\prime p+d, q+d}(V\{W\})$, where $d=\operatorname{dim} V$ $-\operatorname{dim} W$ and $i: W \rightarrow V$ is the inclusion. In particular, $[W]=i_{*}[1]$ $\in \emptyset^{\prime d, d}(V\{W\})$.

Proof. Suppose $f_{1}, \ldots, f_{d}$ are holomorphic functions such that $\left\{f_{1}=\cdots=f_{d}\right.$ $=0\}=W$. Since it will follow from Proposition 2.18 that the form $\eta \wedge \omega^{d-1}$ of Example 1.4 is in $A^{d, d-1}(V\{W\})$, then also

$$
i_{*}[\varphi]=d\left[\eta \wedge \omega^{d-1} \wedge \tilde{\varphi}\right]+\left[\eta \wedge \omega^{d-1} \wedge d \tilde{\varphi}\right]
$$

is in $\Phi^{\prime p+d, q+d}(V\{W\})$, where $\tilde{\varphi}$ is any smooth extension of $\varphi$ to $V$.

Thus we can choose an open cover $\left\{U_{i}\right\}$ of $V$ such that the restriction of $i_{*}[\varphi]$ to each $U_{i}$ is in $\Phi^{\prime p+d, q+d}\left(U_{i}\left\{W \cap U_{i}\right\}\right)$. But then if $\sum \lambda_{i}=1$ is a partition of unity subordinate to $\left\{U_{i}\right\}, \lambda_{i} i_{*}[\varphi]$ is in $\Phi^{\prime p+d q+d}(V\{W\})$ and so is $i_{*}[\varphi]$ $=\sum \lambda_{i} i_{*}[\varphi]$.

The sheaves of germs of currents in $\mathscr{D}^{\prime r}(V\{W\})$ and $\mathscr{D}^{\prime p, q}(V\{W\})$ are $\mathscr{K}_{V\{W\}}^{r}$ and $\mathcal{K}_{V\{w\}}^{p, q}$.

Theorem 2.10. The following sequences are fine resolutions:

$$
\begin{aligned}
& 0 \rightarrow \hat{\Omega}^{p} \rightarrow F^{p} \mathcal{K}_{V\{W\}}^{p} \stackrel{d}{\rightarrow} F^{p K_{V\{W\}}^{p+1}} \stackrel{d}{\rightarrow} \cdots, \\
& 0 \rightarrow \mathbf{C}_{V} \rightarrow \mathcal{K}_{V\{W\}}^{0} \stackrel{d}{\rightarrow} \mathcal{K}_{V\{W\}}^{1} \stackrel{d}{\rightarrow} \cdots, \\
& 0 \rightarrow \Omega^{p} \rightarrow K_{V\{W\}}^{p, 0} \stackrel{d^{\prime \prime}}{\rightarrow} \mathcal{K}_{V\{W\}}^{p, 1} \stackrel{d^{\prime \prime}}{\rightarrow} \cdots .
\end{aligned}
$$

Proof. The exactness will follow from the Poincare and Dolbeault Lemmas 3.21 and 3.1. That the resolution is fine results from Proposition 2.2; and the fact that the kernels are $\hat{\Omega}^{p}$ and $\Omega^{p}$ follows from (2.4).

Theorem 2.13 (Existence of a global residue form). Let $W$ be a submanifold of codimension $d$ in $V$. 
(a) If $\varphi \in F^{p} A^{r}(W)$ and $d \varphi=0, i_{*}[\varphi]=d[\eta]+[\alpha]$ where

$$
\eta \in F^{p+d} A^{2 d+r-1}(V,\{W\}) \text { and } \alpha \in F^{p+d} A^{2 d+r}(V)
$$

(b) If $\psi \in A^{p, q}(W)$ and $d^{\prime \prime} \psi=0, i_{*}[\psi]=d^{\prime \prime}[\rho]+[\beta]$ where

$$
\rho \in A^{p+d, q+d-1}(V\{W\}) \text { and } \beta \in A^{p+d, q+d}(V)
$$

In particular we may take $1=\varphi=\psi \in A^{0}(W)$ and $i_{*}[1]=[W]$.

Proof. This is an immediate corollary of Proposition 2.9 and Theorem 2.10, using the proof of Corollary 2.5 .

To define $A^{r}(V\{W\})$ we will need the procedure of blowing up $V$ along $W$. First we will describe the blowing up of $\mathbf{C}^{n}$ at $\mathbf{0}$.

If $(z)=\left(z_{1}, \ldots, z_{n}\right)$ are linear coordinates in $\mathbf{C}^{n}$, they induce homogeneous coordinates $[z]=\left[z_{1}, \ldots, z_{n}\right]$ in $\mathbf{P}^{n-1}$. If we set $U_{\alpha}=\left\{[z] \in \mathbf{P}^{n-1}: z_{\alpha} \neq 0\right\}$ and $\zeta_{\alpha j}=z_{j} z_{\alpha}^{-1}, j=1, \ldots, n$, then $\zeta_{a 1}, \ldots, \zeta_{a \alpha-1}, \zeta_{\alpha a+1}, \ldots, \zeta_{a n}$ define holomorphic coordinates in $U_{\alpha}$.

The canonical line bundle $\mu: L \rightarrow \mathbf{P}^{n-1}$ is the line bundle associated to the principal bundle $\mathbf{C}^{n}-(0) \rightarrow \mathrm{P}^{n-1}$ given by $(z) \rightarrow[z]$. The manifold $L$ can also be realized as a submanifold $L \subset \mathbf{C}^{n} \times \mathbf{P}^{n-1}$; the defining equations for $L \cap \mathbf{C}^{n}$ $\times U_{\alpha}$ are $z_{i}=z_{\alpha} \zeta_{\alpha i}, i=1, \ldots, \alpha-1, \alpha+1, \ldots, n$.

The projection of $\mathbf{C}^{n} \times \mathbf{P}^{n-1}$ onto $\mathbf{P}^{n-1}$ induces $\mu: L \rightarrow \mathbf{P}^{n-1}$ and the projection onto the first factor induces $\sigma: L \rightarrow \mathbf{C}^{n}$. The map $U_{\alpha} \times \mathbf{C} \rightarrow \mathbf{C}^{n} \times \mathbf{P}^{n-1}$ which takes $\left(\zeta_{\alpha 1}, \ldots, \zeta_{a n}, t_{\alpha}\right)$ into $\left(t_{\alpha} \zeta_{a 1}, \ldots, t_{\alpha} \zeta_{a n}, \zeta_{a 1}, \ldots, \zeta_{a a-1}, \zeta_{a a+1}, \ldots, \zeta_{a n}\right)$ gives $L$ $\subset \mathbf{C}^{n} \times \mathbf{P}^{n-1}$ the line bundle structure. We observe that

$$
\sigma\left(\zeta_{\alpha 1}, \ldots, \zeta_{a n}, t_{\alpha}\right)=\left(t_{\alpha} \zeta_{\alpha 1}, \ldots, t_{\alpha} \zeta_{a \alpha}, \ldots, t_{\alpha} \zeta_{a n}\right)
$$

where $t_{\alpha} \zeta_{\alpha \alpha}=t_{\alpha}$.

Now it is easy to see that $\sigma^{-1}(0) \simeq \mathrm{P}^{n-1}$ is the zero section of $L$, which is $\left\{t_{\alpha}=0\right\}$ in $U_{\alpha} \times \mathbf{C}$. If we set $E=\sigma^{-1}(0), \sigma: L-E \rightarrow C^{n}-(0)$ is biholomorphic. Furthermore, $\sigma$ is a proper map.

Let us write $\hat{\mathbf{C}}^{n}=L$; this is the blow-up of $\mathbf{C}^{n}$ at $\mathbf{0}$. If we wish to blow up $\mathbf{C}^{m} \times \mathbf{C}^{n}$ along $\mathbf{C}^{m} \times(0)$, we just take the product of the identity map and the map above:

$$
\text { o: } \mathbf{C}^{m} \times \mathbf{C}^{n} \rightarrow \mathbf{C}^{m} \times \mathbf{C}^{n}
$$

If $\Delta^{m}, \Delta^{n}$ are polydisks in $\mathbf{C}^{m}, \mathbf{C}^{n}, \Delta^{m} \times \hat{\Delta}^{n}=\sigma^{-1}\left(\Delta^{m} \times \Delta^{n}\right)$ and

$$
\text { б: } \Delta^{m} \times \hat{\Delta}^{n} \rightarrow \Delta^{m} \times \Delta^{n}
$$

is defined.

Finally, it can be shown that this process is invariant under change of 
coordinates. Thus for any complex submanifold $W \subset V$ of codimension $n$, there is a unique manifold $V[W]$, the blow-up of $V$ along $W$, and a proper map $\sigma: V[W] \rightarrow V$ which in local coordinates is identified with the $\sigma$ above.

Set $E=\sigma^{-1}(W)$, a submanifold of $V[W]$ of codimension one; then:

(a) $\sigma=V[W]-E \rightarrow V-W$ is biholomorphic.

(b) $\sigma: E \rightarrow W$ is a holomorphic $\mathbf{P}^{n-1}$ bundle.

We are now ready to begin to define forms of kernel type.

Definition 2.16. Let $W \subset V$ be a complex subvariety of codimension one. A locally summable $(p, q)$-form $\rho$ will be said to be of kernel type or semimeromorphic along $W$ if in an open set $U$ with $W \cap U=\{t=0\}$ for a holomorphic defining function $t$ we can write $\eta=t^{-1} d t \wedge \varphi+\psi$ where $\varphi \in A^{p-1, q}(U), \psi$ $\in A^{p, q}(U)$ are smooth forms. We denote the space of such forms by $A^{p, q}(V\{W\})$. If $W$ is a submanifold, $d t \neq 0$ near $W$. N.B. Any such form is locally summable and defines a current $[\eta] \in \mathscr{D}^{p, q}(V)$. This is clear for nonsingular $W$; see the proof of Proposition 5.3 for the general case.

Definition 2.17. If $W \subset V$ is a complex submanifold, the space of $(p, q)$ forms of kernel type along $W$ is the space of locally summable forms: $A^{p, q}(V\{W\})$ $=\left\{\eta: \sigma^{*} \eta \in A^{p, q}(V[W]\{E\})\right\}$. If codimension $W=1$, this agrees with Definition 2.16 since in this case $\sigma$ is the identity map.

We remark that $\sigma^{*}$ is defined for locally summable forms. Since $\sigma$ is biholomorphic off the (Lebesgue) null sets $W$ and $E$, we can equivalently define

$$
A^{p, q}(V\{W\})=\left\{\sigma_{*}[\eta]: \eta \in A^{p, q}(V[W]\{E\})\right\}
$$

if we consider $A^{p, q}(V\{W\}) \subset \mathscr{D}^{\prime p, q}(V)$. Clearly any such current is a locally summable form.

Proposition 2.18. If $\left(f_{1}, \ldots, f_{r}\right)$ are any holomorphic functions on $V$ of rank $r$ such that the submanifold $W=\left\{f_{1}=\cdots=f_{r}=0\right\}$, then, given the forms of Example 1.4, $\eta=(2 \pi i)^{-1} d^{\prime} \log \left(\left|f_{1}\right|^{2}+\cdots+\left|f_{r}\right|^{2}\right)$ and $\omega=d^{\prime \prime} \eta$ the forms $\eta \wedge \omega^{r}$ and $\omega^{r}$ are of kernel type along $W$ for any $r$.

Proof. Letting $\left(f_{1}, \ldots, f_{r}\right)$ be part of a local coordinate system on $V$, and using (2.14), we see that in $U_{\alpha} \times \mathbf{C}, \sigma^{*} \eta=(2 \pi i)^{-1}\left(t_{\alpha}^{-1} d t_{\alpha}+d^{\prime} \log \left(1+\sum_{i=\alpha}\left|\zeta_{\alpha i}\right|^{2}\right)\right)$. This is clearly of the required form. Furthermore $\sigma^{*} \omega$ $=(2 \pi)^{-1} i d^{\prime} d^{\prime \prime} \log \left(1+\sum_{i \neq \alpha}\left|\zeta_{\alpha i}\right|^{2}\right)$, which is a $C^{\infty}$ form, in fact the Kähler form of the Fubini-Study metric on $\mathbf{P}^{n-1}$. The proposition follows.

3. Dolbeault lemma for currents of residue type. Let $\Delta^{m} \subset \mathbf{C}^{m}$ denote any polydisk centered at the origin.

Proposition 3.1. If $u \in \mathscr{D}^{\prime p, q}\left(\Delta^{m} \times \Delta^{n}\left\{\Delta^{m} \times(0)\right\}\right)$ and $d^{\prime \prime} u=0$, there exists a $v \in \Phi^{\prime p, q-1}\left(\Delta^{m} \times \Delta^{n}\left\{\Delta^{m} \times(0)\right\}\right)$ such that $d^{\prime \prime} v=u$. 
Proof. We will write $\hat{\Delta}^{n}$ instead of $\Delta^{n}[0]$ in the notation of (2.15). If $\sigma: \Delta^{m} \times \hat{\Delta}^{n}$ $\rightarrow \Delta^{m} \times \Delta^{n}$ and $E=\sigma^{-1}\left(\Delta^{m} \times(0)\right) \simeq \Delta^{m} \times \mathbf{P}^{n-1}$, then by definition

$$
u=\sigma_{*}\left[\rho_{0}\right]+d^{\prime} \sigma_{*}\left[\rho_{1}\right]+d^{\prime \prime} \sigma_{*}\left[\rho_{1}\right]
$$

where $\rho_{i} \in A^{p+q-i}\left(\Delta^{m} \times \hat{\Delta}^{n}\{E\}\right)$.

Thus if $\{t=0\}$ is the local equation for $E$, locally $\rho_{1}=t^{-1} d t \wedge \varphi+\psi$. But we see that $d^{\prime}\left[\rho_{1}\right]=\left[d^{\prime} \rho_{1}\right]$, where

$$
d^{\prime} \rho_{1}=-t^{-1} d t \wedge d^{\prime} \varphi+d^{\prime} \psi \in A^{p+q}\left(\Delta^{m} \times \hat{\Delta}^{n}\{E\}\right),
$$

because $d^{\prime}\left[t^{-1} d t\right]=d^{\prime} d^{\prime}\left[\log |t|^{2}\right]=0$.

Thus $u=\sigma_{*}\left[\rho_{0}+d^{\prime} \rho_{1}\right]+d^{\prime \prime} \sigma_{*}\left[\rho_{1}\right]$. Furthermore, the forms in brackets can be assumed to be of type $(p, q)$ and $(p, q-1)$ respectively since the other components cancel out. Since Proposition 3.1 is true trivially for $d^{\prime \prime} \sigma_{*}\left[\rho_{1}\right]$, we may assume henceforth that

$$
u=\sigma_{*}[\rho]
$$

where $\rho \in A^{p, q}\left(\Delta^{m} \times \hat{\Delta}^{n}\{E\}\right)$.

The proof will be broken into three parts, beginning with the hypersurface case.

(a) Hypersurface case. Suppose $n=1$; then $\Delta^{m} \times \hat{\Delta}^{1}=\Delta^{m} \times \Delta^{1}$. If $\left(z_{1}, \ldots, z_{m}\right.$, t) are linear coordinates,

$$
u=\left[(2 \pi i t)^{-1} d t \wedge \varphi+\psi\right]
$$

where $\varphi$ and $\psi$ are $C^{\infty}$ forms with no term involving $d t$ (saying nothing about $d \bar{t}$ ). Then

$$
0=d^{\prime \prime} u=i_{*}\left[i^{*} \varphi\right]-\left[(2 \pi i t)^{-1} d t \wedge d^{\prime \prime} \varphi-d^{\prime \prime} \psi\right] .
$$

Since $d^{\prime \prime} \varphi$ and $d^{\prime \prime} \psi$ have no $d t$ term, both $d^{\prime \prime} \varphi=0$ and $d^{\prime \prime} \psi=0$ on $\Delta^{m} \times \Delta^{1}-\Delta^{m} \times(0)$ and hence on $\Delta^{m} \times \Delta^{1}$ by continuity.

By the usual Dolbeault lemma there exist forms $\varphi_{1}, \psi_{1}$ such that $d^{\prime \prime} \varphi_{1}=\varphi$, $d^{\prime \prime} \psi_{1}=\psi$. If we set $v_{1}=\left[-\left(2 \pi i t^{-1}\right) d t \wedge \varphi_{1}+\psi_{1}\right]$, then

$$
u-i_{*}\left[i^{*} \varphi_{1}\right]=d^{\prime \prime} v_{1} \text {. }
$$

Now $i_{*}\left[d^{\prime \prime} i^{*} \varphi_{1}\right]=d^{\prime \prime} i_{*}\left[i^{*} \varphi_{1}\right]=0$, which implies that as a form on $\Delta^{m} \times(0)$, $d^{\prime \prime} i^{*} \varphi_{1}=0$. We extend $i^{*} \varphi_{1}$ to a closed form $\varphi_{2}$ on $\Delta^{m} \times \Delta^{1}$; for example if $p: \Delta^{m} \times \Delta^{1} \rightarrow \Delta^{m}$ is the projection, let $\varphi_{2}=p^{*} i^{*} \varphi_{1}$. Then if $v_{2}=\left[-\left(2 \pi i t^{-1}\right) d t\right.$ $\left.\wedge \varphi_{2}\right], u=d^{\prime \prime}\left(v_{1}+v_{2}\right)$ since $i_{*}\left[i^{*} \varphi_{2}\right]=i_{*}\left[i^{*} \varphi_{1}\right]$. Since

$$
v_{1}+v_{2} \in \mathscr{D}^{\prime p, q-1}\left(\Delta^{m} \times \Delta^{1}\left\{\Delta^{m} \times(0)\right\}\right),
$$

this proves Proposition 3.1 and hence Theorem 2.13 for submanifolds $W$ of codimension one. 
(b) In order to study the general case, we need the following results:

$$
\sigma^{*}: H^{0}\left(\Delta^{n}, \Omega \rho_{n}\right) \rightarrow H^{0}\left(\hat{\Delta}^{n}, \Omega{\underline{\Delta^{n}}}^{p^{n}}\right)
$$

is an isomorphism.

$$
\begin{aligned}
H^{q}\left(\hat{\Delta}^{n}, \Omega_{\Delta^{n}}^{P}\right) & \simeq \mathrm{C} & & \text { for } 0<p=q<n, \\
& =0 & & \text { for other } q>0 .
\end{aligned}
$$

To prove (3.4) for $n>1$, observe that $\sigma^{-1 *} \varphi$, for any $\varphi \in H^{0}\left(\hat{\Delta}^{n}, \Omega_{\Delta^{n}}\right)$, defines a holomorphic form on $\Delta^{n}-(0)$ which extends to $\Delta^{n}$ by Hartogs' theorem. Thus $\sigma^{-1 *}$ followed by extension is an inverse to $\sigma^{*}$. For $n=1, \sigma^{*}$ is the identity.

We will prove (3.5) by using local cohomology (see [11]) and induction on $n$. The case $n=1$ is trivial.

First we will prove that

$$
H^{q}\left(\hat{\Delta}^{n}, \Omega^{p}\right) \simeq H^{q}\left(\hat{C}^{n}, \Omega^{p}\right) \text { for } q>0 .
$$

We denote by $\Delta^{n^{*}}$ the space $\hat{\Delta}^{n}-E \simeq \Delta^{n}-(0)$ and likewise for $\mathbf{C}^{n^{*}}$. The inclusion $i: \hat{\Delta}^{n} \subset \hat{\mathbf{C}}^{n}$ induces the long exact sequence homomorphism

$$
\begin{aligned}
& \cdots \longrightarrow H_{E}^{q}\left(\hat{\Delta}^{n}, \Omega^{p}\right) \longrightarrow H^{q}\left(\hat{\Delta}^{n}, \Omega^{p}\right) \longrightarrow H^{q}\left(\Delta^{n^{*}}, \Omega^{p}\right) \longrightarrow \cdots \\
& \begin{array}{l}
\uparrow_{q} \quad \oint_{\beta_{q}} \quad \prod_{\gamma_{q}} \\
\ldots \longrightarrow H_{E}^{q}\left(\hat{\mathbf{C}}^{n}, \Omega^{p}\right) \longrightarrow H^{q}\left(\hat{\mathbf{C}}^{n}, \Omega^{p}\right) \longrightarrow H^{q}\left(\mathbf{C}^{n^{*}}, \Omega^{p}\right) \longrightarrow \ldots
\end{array}
\end{aligned}
$$

By (3.4) we break off the $\boldsymbol{H}^{0}$ part and consider the sequence beginning at the $H^{1}$ level. The maps $\alpha_{q}$ are isomorphisms by excision. We show that the $\gamma_{q}$ are isomorphisms by a second exact sequence, which we can also begin at the $H^{\mathbf{1}}$ level:

$$
\cdots \longrightarrow H_{\{0\}}^{q}\left(\Delta^{n}, \Omega^{p}\right) \longrightarrow H^{q}\left(\Delta^{n}, \Omega^{p}\right) \longrightarrow H^{q}\left(\Delta^{n^{*}}, \Omega^{p}\right) \longrightarrow \cdots
$$

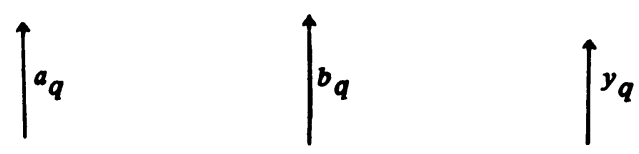

$$
\cdots \longrightarrow H_{\{0\}}^{q}\left(C^{n}, \Omega^{p}\right) \longrightarrow H^{q}\left(\mathbf{C}^{n}, \Omega^{p}\right) \longrightarrow H^{q}\left(\mathbf{C}^{n^{*}}, \Omega^{p}\right) \longrightarrow \cdots
$$

The $a_{q}$ are isomorphisms by excision. The middle cohomology groups are all zero, so $b_{q}$ is an isomorphism. Hence by the Five Lemma and (3.8) the $\gamma_{q}$ are isomorphisms. Applying the Five Lemma to (3.7) we then get (3.6).

Next we compute $H^{q}\left(\mathfrak{C}^{n}, \Omega^{p}\right)$. Let $H$ be the hyperplane at infinity, so that 
$\mathbf{C}^{n}=\mathbf{P}^{n}-H$. Also let $\mathbf{P}^{n}$ be projective space with one point blown up, i.e., $\hat{\mathbf{P}}^{n}=\hat{\mathbf{C}}^{n} \cup H$ in the natural way.

Recall that $\mu: \hat{\mathbf{C}}^{n} \rightarrow \mathbf{P}^{n-1}$ sending $\left(z_{1}, \ldots, z_{n}\right)$ into $\left[z_{1}, \ldots, z_{n}\right]$ gives $\hat{\mathbf{C}}^{n}$ the structure of a holomorphic line bundle. This extends to a map $\mu: \mathbf{P}^{n} \rightarrow \mathbf{P}^{n-1}$ which is a holomorphic fiber bundle with fiber $\mathbf{P}^{\mathbf{l}}$. Also the map $\sigma$ extends to a map o: $\hat{\mathbf{P}}^{n} \rightarrow \mathbf{P}^{n}$.

We will use the following commutative diagram of exact sequences induced by б:

$$
\begin{aligned}
& \left\lceil\sigma ^ { * } ( 1 ) \quad \left\lceil\sigma ^ { * } ( 2 ) \quad \left\lceil\sigma^{*}(3)\right.\right.\right. \\
& \cdots \longrightarrow H_{H}^{q}\left(\mathbf{P}^{n}, \Omega^{p}\right) \stackrel{\alpha_{q}}{\longrightarrow} H^{q}\left(\mathbf{P}^{n}, \Omega^{p}\right) \stackrel{\beta}{\longrightarrow} H^{q}\left(\mathbf{C}^{n}, \Omega^{p}\right) \longrightarrow \cdots
\end{aligned}
$$

Again by excision $\sigma^{*}(1)$ is an isomorphism for all $q$. Also $\sigma^{*}(2)$ is an injection for all $q$. This is easy to see, for the generator of $H^{p}\left(P^{n}, \Omega^{p}\right)$ in Dolbeault cohomology is $\omega^{p}$, where $\omega$ is the Kähler form on $P^{n}$. But the cohomology class of $\sigma^{*} \omega^{p} \neq 0$ since $\int_{p \text { p }} \sigma^{*} \omega^{p} \wedge \sigma^{*} \omega^{n-p} \neq 0$.

Furthermore, since $H^{q}\left(\mathbf{C}^{n}, \Omega^{p}\right)=0$ for $q>0, \alpha_{q}$ is a surjection for $q>0$; in fact $\alpha_{q}$ is an isomorphism for $q>1$. Thus $a_{q}=\sigma^{*}(2) \circ \alpha_{q} \circ \sigma^{*}(1)^{-1}$ is injective for $q>1$, which means $b_{q}$ is surjective for $q>0$.

Since $\hat{\mathbf{P}}^{n}$ is a holomorphic fiber bundle over $\mathbf{P}^{n-1}$ with fiber $\mathbf{P}^{1}$, the Leray spectral sequence shows that $H^{p}\left(\hat{\mathbf{P}}^{n}, \Omega^{p}\right) \simeq \mathbf{C}^{2}$ for $q=1, \ldots, n-1$, and $H^{0}\left(\hat{\mathbf{P}}^{n}, \Omega^{0}\right) \simeq H^{n}\left(\hat{\mathbf{P}}^{n}, \Omega^{n}\right) \simeq \mathrm{C}$. (This sequence degenerates at the $E^{2}$ term by inspection.)

Now the cokernel of $a_{q}$ equals cokernel $\sigma^{*}(2)$ for all $q$ since $\sigma^{*}(1)$ is an isomorphism. Thus for $q>0$, since $b_{q}$ is surjective,

$$
\begin{aligned}
H^{q}\left(\hat{\mathbf{C}}^{n}, \Omega^{p}\right) \simeq \operatorname{Cok} \sigma^{*}(2) & =\mathrm{C} & & \text { if } p=q, p<n, \\
& =0 & & \text { otherwise. }
\end{aligned}
$$

This with (3.6) proves (3.5).

(c) Now we prove the Dolbeault lemma for $\mathscr{D}^{\prime p q q}\left(\Delta^{n}\{0\}\right)$. Let $u=\sigma_{*}[\rho]$, where $\rho \in A^{p, q}\left(\hat{\Delta}^{n}\{E\}\right), q>0$, and suppose $d^{\prime \prime} u=0$.

For any such $\rho, d^{\prime \prime}[\rho]=i_{*}[\varphi]+[\psi]$, where $\psi \in A^{p, q+1}\left(\hat{\Delta}^{n}\{E\}\right)$ and $\varphi$ $\in A^{p-1, q}(E)$, the map $i: E \rightarrow \hat{\Delta}^{n}$ being the inclusion.

But $\sigma_{*}[\psi]=0$ on $\Delta^{n}-(0)$ and thus $\psi=0$, since $\sigma$ is biholomorphic on this set. Thus

$$
d^{\prime \prime}[\rho]=i_{*}[\varphi]
$$

and $i_{*}\left[d^{\prime \prime} \varphi\right]=d^{\prime \prime} i_{*}[\varphi]=d^{\prime \prime} d^{\prime \prime}[\rho]=0$, whence $d^{\prime \prime} \varphi=0$. 
By the Dolbeault cohomology of $E=\mathbf{P}^{n-1}$, there is constant $c$ such that

$$
\varphi=d^{\prime \prime} \lambda+c \omega^{q}
$$

where $\omega_{0}$ is the Kähler form for the Fubini-Study metric on $P^{n-1}$ [21]. Of course $c=0$ for $p \neq q+1$ or (3.11) is meaningless.

Now if $\eta$ and $\omega$ are the forms of Proposition $2.18 \sigma^{*} \omega=\mu^{*} \omega_{0}$, where $\mu: \hat{\Delta}^{n} \rightarrow E$ is the projection, and

$$
d^{\prime \prime}\left(\sigma^{*} \eta \wedge \omega^{q}\right)=i_{*}\left[\omega_{0}^{q}\right]+\left[\sigma^{*} \omega^{q+1}\right]
$$

as is clear from the local expression in Proposition 2.18.

We can write $c \mu^{*} \omega_{g}^{g+1}$ as a boundary:

$$
c\left[\mu^{*} \omega_{0}^{q+1}\right]=d^{\prime \prime}\left(\left[\sigma^{*} c \eta \wedge \omega^{q}\right]+i_{*}[\lambda]-[\rho]\right) .
$$

But on cohomology $\mu^{*}$ is an injective map, since $i^{*} \sigma^{*}$ is the identity. Therefore, if $q \leq n-2, c=0$. But if $q=n-1$, then

$$
0=\sigma_{*} i_{*}[\varphi]=d^{\prime \prime} \sigma_{*} i_{*}[\lambda]+\sigma_{*} i_{*}\left[c \omega_{0}^{n-1}\right]=c,
$$

for $d^{\prime \prime} \sigma_{*} i_{*}[\lambda](\zeta)=\int_{E} \lambda \wedge i^{*} \sigma^{*} d^{\prime \prime} \zeta=0$ because $i^{*} \sigma^{*} d^{\prime \prime} \zeta=0$ on $E$.

Thus we conclude that

$$
d^{\prime \prime}\left([\rho]-i_{*}[\lambda]\right)=0
$$

By (3.5) the injective map

$$
\mu^{*}: H^{q}\left(\mathbf{P}^{n-1}, \Omega_{\mathbf{P}_{n-1}}^{p}\right) \rightarrow H^{q}\left(\hat{\Delta}^{n}, \Omega \mathcal{L}_{n}\right)
$$

is an isomorphism for $q>0$. Furthermore, by part (a) above we have Theorem 2.13 for the codimension one case; thus

$$
H^{q}\left(\hat{\Delta}^{n}, \Omega_{\Delta^{n}}^{P_{n}^{n}}\right) \simeq H^{q}\left(\Gamma K_{\hat{\Delta}^{n}(E)}^{p^{\cdot}}\right)
$$

Therefore,

$$
[\rho]-i_{*}[\lambda]=d^{\prime \prime} v^{\prime}+c\left[\mu^{*} \omega_{0}^{g}\right]
$$

where $v^{\prime} \in \mathscr{D}^{\prime p, q-1}\left(\hat{\Delta}^{n}\{E\}\right), 0<q<n$, and $c=0$ for $p \neq q$.

Now by (3.12),

$$
[\rho]-i_{*}[\lambda]=d^{\prime \prime}\left(v^{\prime}+\sigma^{*} c \eta \wedge \omega^{q-1}\right)-c i_{*}\left[\omega_{0}^{q-1}\right] .
$$

But since $\sigma_{*} i_{*}\left[\omega_{0}^{g-1}\right]=0$ for the same reason that $\sigma_{*} i_{*}[\lambda]=0$, this yields

$$
\sigma_{*}[\rho]=d^{\prime \prime}\left(\sigma_{*} \nu^{\prime}+c \eta \wedge \omega^{q-1}\right) .
$$

This proves Proposition 3.1 for the case $m=0$ and any $n$. To complete the proof one approach would be to observe that $\Omega_{\Delta^{m} \times \Delta^{n}}^{0} \simeq \Omega_{\Delta^{m}}^{0} \hat{\otimes} \Omega_{\Delta^{n}}^{0}$, the topological 
tensor product, and that the resolution (2.12) for $\Omega_{\Delta^{m} \times \Delta^{n}}^{0}$ by $\mathcal{F}_{\Delta^{m} \times \Delta^{n}\left\{\Delta^{m} \times(0)\right\}}^{0, q^{\prime}}$ just the topological tensor product of resolution (2.4)(iii) for $\Omega_{\Delta^{m}}^{0}$ and (2.12) for $\Omega_{\Delta^{n}}^{0}$. Using the Künneth formula for sheaves [17] we conclude Proposition 3.1 for $p=0$. But since the sheaves $\Omega_{\Delta^{m} \times \Delta^{n}}$ and their resolutions are just direct sums of $\Omega_{\Delta^{m}}^{r} \hat{\otimes} \Omega_{\Delta^{n}}^{-r}$ $\simeq \bigoplus \Omega_{\Delta^{m}}^{0} \hat{\otimes} \Omega_{\Delta^{n}}^{p^{-r}}$, the same argument suffices.

A more straightforward approach is to do induction on $m$. This, however, requires that all the results of $\$ 3$ to date be proved under the assumption that all forms of kernel type be allowed to have coefficients which are also $C^{\infty}$ functions of $t \in \Delta^{r}$. This follows from the same proofs using the Dolbeault lemma with parameters (same proof as [6, p. 97]) or even by using topological tensor products [17].

Let $u \in \mathscr{D}^{\prime p, q}\left(\Delta^{1} \times \Delta^{m} \times \Delta^{n}\left\{\Delta^{1} \times \Delta^{m} \times 0\right\}\right)$, where coordinates are $\left(t, z_{1}, \ldots\right.$, $\left.z_{m+n}\right)$. Then $u=u_{0}+d \bar{t} \wedge u_{1}+d t \wedge u_{2}+d t \wedge d \bar{t} \wedge u_{3}$, where each $u_{i}$ involves no $d t$ or $d \bar{t}$ and may thus be considered currents on $\Delta^{m} \times \Delta^{n}$ with parameter $t$ and additional (dummy) parameters $s \in \Delta^{r}$. Now

$$
0=d^{\prime \prime} u=d^{\prime \prime} u_{0}-d \bar{t} \wedge d^{\prime \prime} u_{1}-d t \wedge\left(d^{\prime \prime} u_{2}-d \bar{t} \wedge d^{\prime \prime} u_{3}\right) .
$$

Since the first two terms involve no $d t$ term, $d^{\prime \prime} u_{0}=d \bar{t} \wedge d^{\prime \prime} u_{1}$. If we write $d^{\prime \prime}=d^{\prime \prime}+d_{2}^{\prime \prime}$, then since there is a $d \bar{t}$ factor in $d^{\prime \prime} u_{0}, d_{2}^{\prime \prime} u_{0}=0$. Thus there is a $v_{0}$, a current of $\Delta^{m} \times \Delta^{n}$ with parameters such that $d_{z}^{\prime \prime} v_{0}=u_{0}$. Consequently

$$
u_{0}+d \bar{t} \wedge u_{1}-d^{\prime \prime} v_{0}=d \bar{t} \wedge w_{0}
$$

and $d^{\prime \prime}\left(d \bar{t} \wedge w_{0}\right)=-d \bar{t} \wedge d_{2}^{\prime \prime} w_{0}=0$, where $w_{0}$ involves no $d t$ or $d \bar{t}$. Hence there is a $v_{2}$ with $d_{2}^{\prime \prime} v_{2}=w_{0}$ and $d^{\prime \prime}\left(d \bar{t} \wedge v_{2}\right)=-d \bar{t} \wedge d_{2}^{\prime \prime} v_{2}=-d \bar{t} \wedge w_{0}$. Thus

$$
u_{0}+d \bar{t} \wedge u_{1}=d^{\prime \prime}\left(v_{0}-d \bar{t} \wedge v_{1}\right) .
$$

The same argument applied to $d^{\prime \prime} u_{2}=d \bar{t} \wedge d^{\prime \prime} u_{3}$ gives $u=d^{\prime \prime} v$ and Proposition 3.1 is proved.

Finally we must show the Poincaré lemma to prove (2.11).

Proposition 3.21. Given $u \in F^{p} \mathscr{Q}^{\prime r}\left(\Delta^{m} \times \Delta^{n}\left\{\Delta^{m} \times(0)\right\}\right)$ with $d u=0$, there is a $v \in F^{p \Phi^{\prime r-1}}\left(\Delta^{m} \times \Delta^{n}\left\{\Delta^{m} \times(0)\right\}\right)$ such that $d v=u$, if $r>p$.

Proof. We will work by downward induction, using Proposition 3.1. First we note that Proposition 3.21 is true trivially for $p>n$. Let $u=u_{1}+u_{2}$, where $u_{2}$ is a $(p, r-p)$ current and $u_{1} \in F^{p+1} \mathscr{Q}^{\prime r}$. Then $0=d u=\left(d u_{1}+d^{\prime} u_{2}\right)+d^{\prime \prime} u_{2}$; the $(p, r-p+1)$ component of this form is $d^{\prime \prime} u_{2}=0$. Thus if $r-p>0$ there is a $v^{\prime} \in \Phi^{\prime p, r-p-1}$ such that $d^{\prime \prime} v^{\prime}=u_{2}$. Then $d\left(u-d v^{\prime}\right)=0$ but $u-d v^{\prime}=u_{1}$ $-d^{\prime} v^{\prime} \in F^{p+1} \mathscr{D}^{\prime \prime}$.

By the induction hypothesis, unless $p+1=r$, we can find $v^{\prime \prime} \in F^{p+1} \emptyset^{\prime r-1}$ such that $u=d\left(v^{\prime}+v^{\prime \prime}\right)$. If $p+1=r, u^{\prime}=u-d v^{\prime} \in \Phi^{\prime p+1,0}$. Since $d u^{\prime}=0$, $u^{\prime}$ is a closed holomorphic $(p+1,0)$ form in $\Gamma \hat{\Omega}^{p+1}$ by (2.4)(ii). By the Poincaré 
lemma for holomorphic forms [6, p. 101] there is a form $v^{\prime \prime} \in A^{p, 0}$ such that $d v^{\prime \prime}=u^{\prime}$ and thus $u=d\left(v^{\prime}+v^{\prime \prime}\right)$.

4. Global residue forms for other currents. In this section we construct residue forms for more general currents using kernels on $V \times V$ which are forms of simple kernel type.

For notational purposes it will be useful to extend the definition of $A^{p, q}(V\{W\})$ in a weak way:

Definition 4.1. $A^{p, q}(X\{Y\})$ for complex spaces $Y \subset X$ is the set of locally $L^{1}$ forms $\eta$ on $X$ such that, if $S=\operatorname{Sing} X \cup \operatorname{Sing} Y$, the restriction $\left.\varphi\right|_{X-S}$ $\in A^{p, q}(X-S\{Y-S\})$.

Lemma 4.2. If $W \subset V_{1}$ and $V_{2}$ are all complex manifolds and $f: V_{1} \rightarrow V_{2}$ is a holomorphic map with $\operatorname{rank} f=\operatorname{dim} V_{2}$ and also $\operatorname{rank}(f \mid W)=\operatorname{dim} V_{2}$ as well, then

$$
f_{*}\left(A_{c}^{p+d, q+d}\left(V_{1}\{W\}\right)\right) \subset A^{p, q}\left(V_{2}\right),
$$

where $d=\operatorname{dim} V_{1}-\operatorname{dim} V_{2}$.

Proof. We can assume that $\operatorname{dim} V_{1}=\operatorname{dim} W+1$ since the hypotheses are also satisfied for $V_{1}[W]$. In local coordinates $\left(z_{1}, \ldots, z_{d}, w_{1}, \ldots, w_{r}\right)$ in $U_{\alpha} \subset V_{1}$, and $\left(w_{1}, \ldots, w_{r}\right)$ on $V_{2}$ we may take $W=\left\{z_{1}=0\right\}$ and $f$ the projection on the last $r$ coordinates. If $\varphi \in A_{c}^{p+d q+d}\left(U_{\alpha}\right), \varphi=d z_{1} / z_{1} \wedge \varphi_{1}+\varphi_{2}, \varphi_{i} C^{\infty}$, and $f_{*} \varphi$ at $w$ is $\int_{C^{d}=\left\{\left(z_{1}, \ldots, z_{d}\right)\right\}} d z_{1} / z_{1} \wedge \varphi_{1}(z, w)+\varphi_{2}(z, w)$ which is clearly $C^{\infty}$ in $w$. We may assume supp $\varphi \subset U_{\alpha}$ for some $\alpha$ by using a partition of unity.

Suppose $V_{1}$ and $V_{2}$ are complex manifolds of dimension $n_{1}$ and $n_{2}$. If $p_{i}: V_{1} \times V_{2} \rightarrow V_{i}$ are the projections, then every current $u \in \mathscr{\Phi}^{p, q}\left(V_{1} \times V_{2}\right)$ defines a continuous linear map

$$
T_{u}: A_{c}^{r, s}\left(V_{1}\right) \rightarrow \Phi^{\prime p+r-n_{1}, q+s-n_{1}}\left(V_{2}\right)
$$

by setting

$$
T_{u} \varphi(\psi)=u\left(p_{1}^{*} \varphi \wedge p_{2}^{*} \psi\right)
$$

for $\varphi \wedge A_{c}^{r, s}\left(V_{1}\right)$ and $\psi \in A_{c}^{a, b}\left(V_{2}\right)$ where $a=n_{1}+n_{2}-p-r, b=n_{1}+n_{2}-q$ $-s$.

We also define the adjoint by the same method:

$$
T_{u}^{t} \psi(\varphi)=u\left(p_{1}^{*} \varphi \wedge p_{2}^{*} \psi\right)
$$

which gives $T_{u}^{t}: A_{c}^{a, b}\left(V_{2}\right) \rightarrow \Phi^{\prime p+a-n_{2}, q+b-n_{2}}\left(V_{2}\right)$.

Note that if $p_{2}$ : supp $u \rightarrow V_{2}$ is proper, $T_{u}$ may be extended to $A^{r, s}\left(V_{1}\right)$ and similarly for $T_{u}^{t}$. For special $u$ we may hope to extend $T_{u}$ to all of $\mathscr{D}^{\prime \prime, s}\left(V_{1}\right)$; this is equivalent to proving certain smoothness properties of $T_{u}^{t} \psi$. We proceed to do this when $u$ is given by an appropriate form of kernel type; the problem will be reduced to the smoothing properties for the classical kernel for $d^{\prime \prime}$ on C. 
Lemma 4.5. Let $V$ be a complex manifold of dimension $m$ and let $u=[\eta]$ for $\eta \in A_{c}^{p, q}(V \times V\{\Delta\})$, where $\Delta \subset V \times V$ is the diagonal. Then, writing $T_{\eta}$ for $T_{[\eta]}$,

(i) $T_{\eta}^{t} \psi$ is $C^{\infty}$ for all $C^{\infty} \psi$, i.e., $T_{\eta}^{t}\left(A^{r, s}(V)\right) \subset A^{m-a, m-b}(V) \subset \Phi^{\prime m-a, m-b}(V)$, where as above $a=2 m-p-r, b=2 m-q-s$.

(ii) Let $\psi_{1}, \psi_{2}, \ldots$ be a sequence in $A_{c}^{r, s}(V)$ all supported in the same compact $K \subset U$ an open coordinate neighborhood such that there is a uniform bound for the absolute values of all the coefficient functions of all the $\psi_{i}$. Then if the $\psi_{i}$ converge to zero almost everywhere, then the sequence $T_{\eta}^{t} \psi_{i}$ converges uniformly in a $C^{0}$ sense, i.e., the coefficient functions converge uniformly on compacts, and there is a compact $K^{\prime}$ with supp $T_{\eta}^{t} \psi_{i} \subset K^{\prime}$ for all $i$.

Proof. Part (i) follows from Lemma 4.2. We will reduce (ii) to the case where $V=\mathbf{C}^{m}$. First cover $V$ by a locally finite family $\left\{U_{\alpha}\right\}$ of coordinate neighborhoods; then $\left\{U_{\alpha} \times U_{\alpha}, V \times V-\Delta\right\}$ is a locally finite open cover of $V \times V$. Let $\left\{\lambda_{\alpha}, \lambda\right\}$ be a partition of unity with respect to this cover (supp $\left.\lambda \subset V \times V-\Delta\right)$; then $\lambda \eta$ is a $C^{\infty}$ form in $A_{c}^{p, q}(V \times V)$ and (ii) will be shown at the end of the proof for $C^{\infty} \eta$. Since $\eta=\lambda \eta+\sum_{\alpha} \lambda_{\alpha} \eta$ it suffices to check (ii) for $\lambda_{\alpha} \eta$, which can be considered to be in $A_{c}^{p, q}\left(\mathbf{C}^{m} \times \mathbf{C}^{m}\{\Delta\}\right)$. We will write $\eta$ instead of $\lambda_{\alpha} \eta$ below.

The proof of (ii) depends on the fact that in $\mathbf{C}$

$$
\lim _{\delta \rightarrow 0} \int_{|t| \leq \delta} t^{-1} d t \wedge d \bar{t}=0
$$

We observe that from (i), if $(z, w)$ are coordinates in $\mathbf{C}^{m} \times \mathbf{C}^{m}, T_{\eta}^{t} \psi_{i}=\left[\alpha_{i}\right], \alpha_{i}$ $\in A_{c}^{m-a, m-b}\left(\mathbf{C}^{m}\right)$ with $\alpha_{i}(z)=\int_{z \times C^{m}} \eta \wedge p_{2}^{*}\left(\psi_{i}\right)$, the integration with respect to the $d w$.

First we claim that there is a $C^{\infty}$ function $0 \leq \mu \leq 1$ with $\mu=1$ in a neighborhood of $\Delta$ defined for any $\varepsilon>0$ such that

$$
\left\|\int_{z \times \mathrm{C}^{m}} \mu \eta \wedge p_{2}^{*} \psi_{i}\right\|_{z}<\varepsilon / 2 \text { for all } z,
$$

where \|\|$_{z}$ means the maximum of the coefficient functions at $z$.

We prove this by lifting to get $\sigma^{*}\left(\eta \wedge p_{2}^{*} \psi_{i}\right)$ on $\mathbf{C}^{m} \times \mathbf{C}^{m}[\Delta]$. We break this form up by a partition of unity subordinate to the $m$ coordinate neighborhoods $\left(z_{1}, \ldots, z_{m}, \zeta_{1}, \ldots, \zeta_{m-1}, t\right)$ with $\{t=0\}=E=\sigma^{-1}(\Delta)$ and $p_{1} \circ \sigma(z, \zeta, t)=(z)$, cf. 2.14. Now we have a finite sum of integrals of the form

$$
I(R)=\int_{|t|^{2}+\Sigma\left|\zeta_{i}\right|^{2}<R} f_{i}(z, \zeta, t) t^{-1} d t d \bar{t} d \zeta_{1} d \bar{\zeta}_{1} \cdots d \zeta_{m-1} d \bar{\zeta}_{m-1} \text {. }
$$

By assumption the $C^{\infty}$ functions $f_{i}$ are uniformly bounded, so for some $\delta>0$, $|I(R)|<\varepsilon / 2 m$ for $r<\delta$. Now choose any $\mu$ which is supported in $\sigma$ (union of the $\delta$ neighborhoods).

Now $\eta=\mu \eta+(1-\mu) \eta$ and $(1-\mu) \eta$ is $C^{\infty}$. If we know (ii) for $C^{\infty} \eta$ we are done, for then we see that for large $i,\left\|\int_{z \times C^{m}}(1-\mu) \eta \wedge p_{2}^{*} \psi_{i}\right\|_{z}<\varepsilon / 2$ and thus $\left\|\alpha_{i}(z)\right\|_{z}<\varepsilon$, which will end the proof. 
To show (ii) for $\eta$ smooth, we can again reduce to $V=\mathbf{C}^{m}$. We observe that $\left\|\int_{z \times C^{m}} \eta \wedge p^{*} \psi_{i}\right\|_{z}<M C_{i}$ where $M$ depends on the supremum over $\mathbf{C}^{m} \times \mathbf{C}^{m}$ of the coefficients of $\eta$ and $C_{i}$ is the maximum of the integrals of the coefficients of $\psi_{i}$ over $\mathbf{C}^{m}$. By hypothesis and the bounded convergence theorem, $C_{i} \rightarrow 0$ as $i \rightarrow \infty$.

A current $u \in \mathscr{D}_{r}^{\prime}(V)$ can be considered to be a form whose coefficients are distributions; a current of order zero is a form whose coefficients are Radon measures. Equivalently, a current $u$ is of order zero if $u$ can be extended to a continuous linear functional

$$
\text { u: } C^{0} A_{c}^{r}(V) \rightarrow \mathrm{C}
$$

on the space of continuous $r$-forms with convergence of $\left\{\varphi_{i}\right\}$ defined to be uniform convergence of coefficients on a compact set $K$ such that supp $\varphi_{i} \subset K$ for all $i$ (see [29, p. 25]).

Proposition 4.7. Given $\eta \in A^{p, q}(V \times V\{\Delta\})$ such that $p_{2}$ : supp $\eta \rightarrow V$ is proper, then

(a) the map $T_{\eta}$ extends to a continuous map $T_{\eta}: \mathscr{D}^{\prime r, s}(V) \rightarrow \mathscr{Q}^{\prime r+p-m, s+q-m}(V)$;

(b) for any current $u$, sing supp $T_{\eta} u \subset \operatorname{sing} \operatorname{supp} u$;

(c) if $u$ is of order zero, $T_{\eta} u=[\xi]$, where $\xi$ is a form with locally $L^{1}$ coefficients.

Proof. The extension of $T_{\eta}$ is defined by $T_{\eta} u(\psi)=u\left(T_{\eta}^{t} \psi\right)$, which is well defined by Lemma 4.5(i). To prove the continuity of $T_{\eta}$ reduces to proving the continuity of $T_{\eta}^{t}: A_{c}^{r, s}(V) \rightarrow A_{c}^{m-a, m-b}(V)$, which is clear from local expressions of the form of the integrand of $(4.6)$ (cf. [29, p. 105]).

Statement (b) is also immediate from Lemma 4.5(i). Suppose $x \notin$ sing supp $u$; let $0 \leq \lambda \leq 1$ be a $C^{\infty}$ function with $\lambda \equiv 1$ near $x$ and supp $\lambda \cap$ sing supp $u$ $=\varnothing$. Then $u=\lambda u+(1-\lambda) u$. Since $\lambda u$ is $C^{\infty}$, so is $T_{\eta} \lambda u$. Now $T_{\eta}(1-\lambda) u$ $=T_{\left(1-\lambda \circ p_{1}\right) \eta} u$ by definition, and $\left(1-\lambda \circ p_{1}\right) \eta$ is $C^{\infty}$ in $p_{2}^{-1}(U)$, where $U$ is any open set on which $\lambda \equiv 1$ (because sing $\operatorname{supp}\left(1-\lambda \circ p_{1}\right) \eta \subset \Delta \cap$ $\left.\operatorname{supp} p_{2}^{-1}(1-\lambda)\right)$. Thus in $U, T_{\eta}(1-\lambda) u=u_{z}\left(\left(1-\lambda \circ p_{1}\right) \eta(z, w)\right)$, that is $u$ applied to the $z$-part of the form. This is $C^{\infty}[29$, p. 105].

Part (c) follows from Lemma 4.5(ii) which can be interpreted as saying that $T_{\eta}^{t}$ is a continuous map from forms with compact support and $L^{\infty}$ coefficients to forms with compact support with $C^{0}$ coefficients. Dualizing we get that $T_{\eta}$ is a map from forms with Radon measure coefficients to forms with locally $L^{1}$ coefficients.

Lemma 4.8. If $v \in \mathscr{D}^{\prime r}(V \times V)$,

$$
\begin{aligned}
T_{d^{\prime \prime}}^{t} & =d^{\prime \prime} \circ T_{\nu}^{t} \circ \varepsilon+\varepsilon \circ T_{\nu}^{t} \circ d^{\prime \prime}, \\
T_{d}^{t} & =d \circ T_{\nu}^{t} \circ \varepsilon+\varepsilon \circ T_{\nu}^{t} \circ d^{\prime \prime},
\end{aligned}
$$

where $\varepsilon(u)=(-1)^{s} u$ for $u \in \Phi^{\prime s}(V)$. Consequently, if $\eta \in A^{r}(V \times V\{\Delta\})$ and 
$p_{2}$ : supp $\eta \rightarrow V$ is proper, then $T_{d^{\prime \prime}[\eta]}$ and $T_{d[\eta]}$ extend from $A^{\prime}(V)$ to $\mathscr{D}^{\prime \prime}(V)$ by the formulas:

$$
\begin{aligned}
T_{d^{\prime \prime}[\eta]} & =\varepsilon \circ T_{\eta} \circ d^{\prime \prime}+d^{\prime \prime} \circ T_{\eta} \circ \varepsilon, \\
T_{d[\eta]} & =\varepsilon \circ T_{\eta} \circ d+d \circ T_{\eta} \circ \varepsilon .
\end{aligned}
$$

Proof. The second formulas do define mappings on $\mathscr{D}^{\prime \prime}(V)$ by Proposition 4.7; one proves it is an extension of $T_{d^{\prime \prime \eta \eta]}}$, etc., by taking adjoints of the first formulas. To see the first ones, note that

$$
\begin{aligned}
T_{d^{\prime v} v}^{t} \psi(\varphi) & =d^{\prime \prime} v\left(p_{1}^{*} \varphi \wedge p_{2}^{*} \psi\right) \\
& =(-1)^{r+1}\left(v\left(p_{1}^{*} d^{\prime \prime} \varphi \wedge p_{2}^{*} \psi\right)+(-1)^{s} v\left(p_{1}^{*} \varphi \wedge p_{2}^{*} d^{\prime \prime} \psi\right)\right) \\
& =(-1)^{r+1}\left(T_{v} \psi\left(d^{\prime \prime} \varphi\right)+(-1)^{s} T_{\nu} d^{\prime \prime} \psi(\varphi)\right) \\
& =\left(d^{\prime \prime} \circ T_{v} \circ \varepsilon(\psi)+\varepsilon \circ T_{v} \circ d^{\prime \prime}(\psi)\right)(\varphi), \text { etc. } \square
\end{aligned}
$$

Given an open neighborhood $N$ of the diagonal $\Delta \subset V \times V$, we let $\Phi(N)$ be the family of closed sets $F \subset N$ such that $p_{i}: F \rightarrow V, i=1,2$, is proper. This $\Phi(N)$ is a family of supports, and we can consider the forms $A_{\Phi}^{p, q}(V \times V)$ and currents $\mathscr{D}_{\Phi}^{\prime}(V \times V)$, etc., which are forms and currents $u$ such that supp $u \in \Phi$. (We will let $N$ be understood.) In particular, for any such $N, \Delta \subset \Phi$ and $[\Delta] \in \mathscr{D}_{\Phi}^{\prime m, m}(V \times V\{\Delta\})$. We can compute the sheaf cohomology with supports in $\Phi, H_{\Phi}^{r}\left(V \times V, \hat{\Omega}^{p}\right)$ and $H_{\Phi}^{q}\left(V \times V, \Omega^{p}\right)$ by means of the resolutions (2.11) and

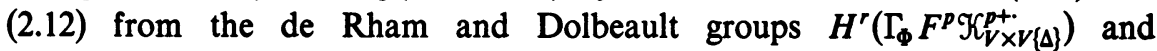
$H^{q}\left(\Gamma_{\Phi} \mathcal{K}_{V \times V(\Delta)}^{p \cdot}\right)(\operatorname{see}[9$, p. 181]).

In particular, we can write

$$
\begin{aligned}
& {[\Delta]=d^{\prime \prime}[\eta]+[\xi],} \\
& {[\Delta]=d[\alpha]+[\beta],}
\end{aligned}
$$

where $\eta \in A_{\Phi}^{m, m-1}(V \times V\{\Delta\}), \xi \in A_{\Phi}^{m, m}(V \times V), \alpha \in F^{m} A_{\Phi}^{2 m-1}(V \times V\{\Delta\}), \beta$ $\in F^{m} A_{\Phi}^{2 m}(V \times V)$.

We now use these kernels to provide a global residue formula for any current $u$ which has special properties for certain $u$. The results of this section are collected in the theorem below. Parts (a), (b) and (c) of this theorem are essentially standard results about singular integral operators, but (4.12) is a more delicate result.

Theorem 4.11 (Global residue formula). Given any current $u \in \mathscr{D}^{\prime r, s}(V)$, using the notation of (4.9) and (4.10),

$$
\begin{aligned}
& u= \pm d^{\prime \prime} T_{\eta} u \pm T_{\eta} d^{\prime \prime} u+T_{\xi} u, \\
& u= \pm d T_{\alpha} u \pm T_{\alpha} d u+T_{\beta} u,
\end{aligned}
$$

where the signs are determined by Lemma 4.8 . 
(a) $T_{\xi} u$ and $T_{\beta} u$ are $C^{\infty}$ forms which vary continuously in the $C^{\infty}$ topology as $u$ varies in the distribution topology.

(b) If $u, d^{\prime \prime} u$, and/or du are currents of order zero, then $T_{\eta} u$ and $T_{\alpha} u, T_{\eta} d^{\prime \prime} u$, and/ or $T_{\alpha} d u$ are locally $L^{1}$ forms which vary continuously in the local $L^{1}$ topology as the corresponding currents vary in the order zero topology.

(c) Given any neighborhood $U \supset$ supp $u$, the forms $\eta$ and $\alpha$ can be chosen so that all the currents in (4.12) and (4.13) have support in $U$. Also sing supp $T_{\eta} u$ $\subset$ sing supp $u$, sing supp $T_{\eta} d^{\prime \prime} u \subset \operatorname{sing} \operatorname{supp} d^{\prime \prime} u$, etc.

Proof. The proofs have mostly been given above.

(a) $T_{\xi} u$ is given by applying the current $u$ to the first variable of the smooth two-variable form $\xi$, cf. [29, p. 105].

(b) follows from Proposition 4.7; the continuity statement is implicit in the proof of that theorem.

(c) Choose $N \subset V \times V$ to be a neighborhood of $\Delta$ such that supp $u \times V \cap N$ $\subset V \times U$. Then use this $\Phi(N)$ to choose the forms in (4.9) and (4.10). The sing supp assertion is Proposition 4.7(b).

Theorem 4.12. For any $k$-dimensional subvariety $Y \subset V$, given $\varphi \in A^{r}(Y)$ and $\eta \in A^{s}(V \times V[\Delta])$ with $p_{i}: \operatorname{supp} \eta \rightarrow V$ proper, $i=1,2$, the current $T_{\eta} i_{*}[\varphi]$ is the current defined by a form in $A^{r+s-2 k}(V\{Y\})(i: Y \rightarrow V$ is the inclusion).

Proof. The current $T_{\eta} i_{*}[\phi]$ is an $L^{1}$ form, $C^{\infty}$ on $V-Y$ by Theorem 4.11. To prove the rest this can be reduced to the case that $V=C^{n}$. Given any $x \in V-\operatorname{sing} Y$ and any compactly supported $C^{\infty}$ function $\lambda$ such that $\lambda \equiv 1$ near $x$, let $\lambda_{i}=\lambda \circ p_{i}$ on $V \times V$. Then, for any current $u, T_{\eta} u=\lambda T_{\eta} \lambda^{2} u$ $+\lambda T_{\eta}\left(1-\lambda^{2}\right) u+(1-\lambda) T_{\eta} u$. The last term $=0$ near $x$, and the second is $C^{\infty}$ by (c) of Theorem 4.11. The term $\lambda T_{\eta} \lambda^{2} u=T_{\lambda_{1} \lambda_{2} \eta} \lambda u$. Thus given any coordinate neighborhood $U$ of $x$, if we choose $\lambda$ with supp $\lambda \subset U$, we have supp $\lambda u \subset U$, supp $\lambda_{1} \lambda_{2} \eta \subset U \times U$ and we may transfer these to $\mathbf{C}^{n}$ and $\mathbf{C}^{n} \times \mathbf{C}^{n}$ by the local coordinate functions.

Since $Y$ is nonsingular we may suppose that $Y=0 \times \mathbf{C}^{k} \subset \mathbf{C}^{n-k} \times \mathbf{C}^{k}$. Thus $V[Y]=\mathbf{C}^{n-k} \times \mathbf{C}^{k}$. Also if $p_{2}: \mathbf{C}^{k} \times \mathbf{C}^{n} \rightarrow \mathbf{C}^{n}$, then $T_{\eta} i_{*}[\varphi]=p_{2 *}\left[i^{*} \eta \wedge \varphi\right]$.

Let $(x, y, z)$ be linear coordinates in $\mathbf{C}^{k} \times \mathbf{C}^{n-k} \times \mathbf{C}^{k}$ and let the linear map given by $L(x, y, z)=(x-z, y, z)$ take $\Delta^{\prime}=\Delta \cap C^{k} \times C^{n-k} \times C^{k}$ onto $0 \times 0$ $\times C^{k}$. Also $p_{2} \circ L=p_{2}$.

It is easy to see that if $\sigma: \mathbf{C}^{n} \times \mathbf{C}^{n}[\Delta] \rightarrow \mathbf{C}^{n} \times \mathbf{C}^{n}$ is the monoidal transform the manifold $\sigma^{-1}\left(\mathbf{C}^{k} \times \mathbf{C}^{n}\right)$ is biholomorphic to $\mathbf{C}^{k} \times \mathbf{C}^{n}\left[\Delta^{\prime}\right]$. This is biholomorphic to $\mathbf{C}^{n} \times \mathbf{C}^{k}$ by a unique map that covers $L$.

Thus it suffices to prove the following case:

$$
\mu \in A_{c}^{r+s}\left(\mathbf{C}^{k} \times \mathbf{C}^{n-k} \times \mathbf{C}^{k}\left\{0 \times 0 \times \mathbf{C}^{k}\right\}\right)
$$

and

$$
p_{2}: \mathbf{C}^{k} \times \mathbf{C}^{n-k} \times \mathbf{C}^{k} \rightarrow \mathbf{C}^{n-k} \times \mathbf{C}^{k}
$$


We wish to show that for

$$
\sigma_{0}: \hat{\mathbf{C}}^{n-k} \times \mathbf{C}^{k} \rightarrow \mathbf{C}^{n-k} \times \mathbf{C}^{k}, \quad \sigma_{0}^{*} p_{2 *} \mu \in A_{c}^{r+s}\left(\hat{\mathbf{C}}^{n-k} \times \mathbf{C}^{k}\left\{E \times \mathbf{C}^{k}\right\}\right) .
$$

(We write $p_{2 *} \mu$ for the form that defines the current $p_{2 *}[\mu]$.)

Look at the following commutative diagram:

$$
\begin{array}{cc}
\mathbf{C}^{k} \times \hat{\mathbf{C}}^{n-k} \times \mathbf{C}^{k} \stackrel{\sigma_{1}}{\longrightarrow} \mathbf{C}^{k} \times \mathbf{C}^{n-k} \times \mathbf{C}^{k} \\
\downarrow_{\pi_{2}} & \downarrow^{p_{2}} \\
\hat{\mathbf{C}}^{n-k} \times \mathbf{C}^{k} & \stackrel{\sigma_{0}}{\longrightarrow} \mathbf{C}^{n-k} \times \mathbf{C}^{k}
\end{array}
$$

where $\pi_{2}$ is the projection and the $\sigma_{i}$ are the monoidal transforms. If we can show that $\sigma_{1}^{*} \mu \in A_{c}^{r+s}\left(\mathbf{C}^{k} \times \hat{\mathbf{C}}^{n-k} \times \mathbf{C}^{k}\left\{\mathbf{C}^{k} \times E \times \mathbf{C}^{k}\right\}\right)$ we are done. For then if $t=0$ locally defines $E \times \mathbf{C}^{k} \subset \mathbf{C}^{n-k} \times \mathbf{C}^{k}$, then $\sigma_{1}^{*} \mu=\left(\pi_{2}^{*}\left(t^{-1} d t\right)\right) \wedge \psi_{1}+\psi_{2}$, with $\psi_{t}$ smooth, and $\pi_{2 *} \sigma_{1}^{*} \mu=t^{-1} d t \wedge \pi_{2 *} \psi_{1}+\pi_{2 *} \psi_{2}$ which is in

$$
A^{r+s-2 k}\left(\hat{\mathbf{C}}^{n-k} \times \mathbf{C}^{k}\left\{E \times \mathbf{C}^{k}\right\}\right) \text {. }
$$

But $\pi_{2 *} \sigma_{1}^{*} \mu=\sigma_{0}^{*} p_{2 *} \mu$ since the $\sigma_{i}$ are biholomorphic except on a set of measure zero.

To show that $\sigma_{1}^{*} \mu$ is semimeromorphic, it suffices to factor $\sigma_{1}$ through $\mathbf{C}^{n} \times \mathbf{C}^{k} \simeq \mathbf{C}^{n} \times \mathbf{C}^{k}\left[\Delta^{\prime}\right]$. The injective mapping $j: \mathbf{C}^{k} \times \mathbf{C}^{n-k} \times \mathbf{P}^{n-k-1} \rightarrow \mathbf{C}^{k}$ $\times \mathbf{C}^{n-k} \times \mathbf{P}^{n-1}$ given by $j(x, y,[w])=(x, y,[0, w])$ induces a map $j_{1}: \mathbf{C}^{k} \times \hat{\mathbf{C}}^{n-k}$ $\rightarrow \hat{C}^{n}$, thus $\sigma_{1}=\sigma \circ\left(j_{1} \times 1\right)$. Since $\sigma^{*} \mu$ is of the form $t^{-1} d t \wedge \varphi_{1}+\varphi_{2}$, so is $\left(j_{1} \times 1\right)^{*} \sigma^{*} \mu$.

5. Further properties of residue forms. In this section we establish further properties of the residue forms of the previous section. The most striking property is that the pull-back of such a form is locally integrable; a property not shared by most locally integrable forms.

(a) Properties under $f_{*}$.

Proposition 5.1. If $f: X_{1} \rightarrow X_{2}$ is a holomorphic map of complex spaces, $X_{1}$ is irreducible, and $\eta$ is a compactly supported $r$-form on $X_{1}$ with $L$ coefficients, then $f_{*}[\eta]$ is also a current defined by an $L^{1}$ form, provided that $f\left(X_{1}\right)$ contains an open set.

Proof. The map $f$ has maximal rank except on a set of measure zero in $X_{1}$. Therefore, by the implicit function theorem the set $f^{-1}(B)$ has measure zero in $X_{1}$ if $B$ has measure zero in $X_{2}$. Thus the measure $f_{*}[\eta]$ is absolutely continuous with respect to the volume measure on $X_{2}$.

Proposition 5.2. Let $f: X_{1} \rightarrow X_{2}$ be a proper holomorphic map of complex spaces, $X_{1}$ irreducible. If $\eta \in A_{c}^{p, q}\left(X_{1}\{Y\}\right)$ and if $f\left(\operatorname{Sing} X_{1} \cup\right.$ Sing $\left.Y\right) \neq f\left(X_{1}\right)$, then there is a proper subvariety $Y^{\prime} \subset f\left(X_{1}\right)$ such that $f_{*}[\eta]$ is $C^{\infty}$ on $f\left(X_{1}\right)-Y^{\prime}$. 
Proof. Let

$$
\begin{aligned}
& S_{1}=\left\{x \in \operatorname{Reg} X_{1}: \operatorname{rank} d f_{x}<\operatorname{dim} f\left(X_{1}\right)\right\}, \\
& S_{2}=\left\{x \in \operatorname{Reg} Y: \operatorname{rank} d(f \mid Y)_{x}<\operatorname{dim} f\left(X_{1}\right)\right\} .
\end{aligned}
$$

Then $S=S_{1} \cup S_{2} \cup \operatorname{Sing} X_{1} \cup \operatorname{Sing} Y_{1}$ is a proper subvariety of $X_{1}$. By hypothesis, by Sard's theorem, and by the proper mapping theorem [12], $f(S)$ is a proper subvariety of $f\left(X_{1}\right)$. Let $Y^{\prime}=f(S)$. On $f\left(X_{1}\right)-Y^{\prime} \subset \operatorname{Reg} f\left(X_{1}\right), f_{*}[\eta]$ is $C^{\infty}$ by Lemma 4.2.

Note. We will write $f_{*} \eta$ for the $L^{1}$ form $\psi$ on $X_{2}$ such that $f_{*}[\eta]=[\psi]$.

(b) Properties under $\mathrm{g}^{*}$.

Proposition 5.3. Let $f: V \rightarrow X$ be a proper holomorphic map of a connected complex manifold into a complex space. If $Y \subset V$ is a divisor with normal crossings, there exists a proper subvariety $Y^{\prime} \subset f(V)$ with the following property: for any holomorphic map $g: Z \rightarrow X$ of an irreducible complex space $Z$ such that $f(Z) \oplus Y^{\prime}$ and for any $\eta \in A_{c}^{p, q}(V\{Y\})$ which is semimeromorphic, the form $g^{*} f_{*} \eta$ has locally $L$ coefficients on $Z$.

Proof. Let $Y^{\prime}=f\left(S_{1} \cup S_{2}\right)$, where $S_{1}=\left\{x \in V\right.$ : rank $\left.d f_{x}<\operatorname{dim} f(V)\right\}, S_{2}$ $=\left\{x \in V\right.$ : for some germ of a component $Y_{i} \subset Y$, rank $\left.d\left(f \mid Y_{i}\right)_{x}<\operatorname{dim} f(V)\right\}$. Then, as in Proposition 5.2, $f_{*} \eta$ is $C^{\infty}$ on $f(V)-Y^{\prime}$.

We form the fiber product $W \subset Z \times V: W=\{(z, x) \in Z \times V: g(z)=f(x)\}$. Now let $p_{Z}: W \rightarrow Z$ and $p_{V}: W \rightarrow V$ be the projections. Since locally $\eta$ $=d t / t \wedge \varphi_{1}+\varphi_{2}, p_{V}^{*} \eta=p_{V}^{*} d t / t \wedge p_{V}^{*} \varphi_{1}+p_{V}^{*} \varphi_{2}$ has the same type of singularity.

We observe that on any complex space $W$, if $h$ is a holomorphic function from $W$ to $\mathbf{C}$ which is not constant on any component of $W$, then $d h / h$ is locally $L 1$. A proof follows from resolving the singularities of $W$ by $\sigma: W \rightarrow W$ in such a way that $D=\{h \circ \sigma=0\}$ is a divisor with normal crossings. The set $D=\left\{z_{1}, \ldots, z_{k}\right.$ $=0\}$ in local coordinates and hence $h \circ \sigma=a z_{1}^{n_{1}}, \ldots, z_{k}^{n_{k}}, a$ being a unit. Then $\sigma^{*}(d h / h)=d a / a+\sum n_{i} d z_{i} / z_{i}$, which is locally $L^{1}$ on $\hat{W}$. Since $\hat{W}$ modifies $W$ on a set of measure zero, $h^{-1} d h=\sigma_{*} \sigma^{*} h^{-1} d h$, which is locally $L^{1}$ by Proposition 5.1.

Thus also by Proposition 5.1, the form $p_{W^{*}} p_{V}^{*} \eta$ is locally $L^{1}$ on $Z$. We must verify that $g^{*} f_{*} \eta=p_{W *} p_{V}^{*} \eta$ almost everywhere. This is trivially so on $g^{-1}\left(f(V)-Y^{\prime}\right)$ because $f$, having maximal rank, gives $V-f^{-1}\left(Y^{\prime}\right)$ a local product structure. Since by hypothesis $g^{-1}\left(Y^{\prime}\right)$ has measure zero on $Z$ we are done.

Proposition 5.4. Given a complex manifold $V$, let $\eta \in A^{p, q}(V \times V\{\Delta\})$ such that $p_{i}: \operatorname{supp} \eta \rightarrow V$ is proper, $i=1,2$. Let $i: X \rightarrow V$ be a subvariety and let $g$ be any holomorphic map $g: Z \rightarrow V$ such that $Z$ is irreducible and $f(Z) \oplus X$; then for any $\varphi \in A^{r, s}(X)$, the form $g^{*} T_{\eta} i_{*}[\varphi]$ is locally $L^{1}$ on $Z$.

Remark. Recalling (4.3), we see that if $j: X \times V \rightarrow V \times V$ is the inclusion and $p_{2}: X \times V \rightarrow V$ the projection, $T_{\eta} i_{*}[\varphi]=p_{2 *}\left[j^{*} \eta \wedge \varphi\right]$, because the form $j^{*} \eta$ $\wedge \varphi$ is locally $L^{1}$ on $X \times V$ by Proposition 5.3. 
Proof. The proof is similar to that of Proposition 5.3; we outline it here. Let $Y=\sigma^{-1}(X \times V)$ where $\sigma: V \times V[\Delta] \rightarrow V \times V$. Then $\eta^{\prime}=\sigma^{*}\left(\eta \wedge p_{1}^{*} \varphi\right)$ has poles like $t^{-1} d t \wedge \psi_{1}+\psi_{2}$ on $Y$. Take the fiber product $W \subset Z \times Y: W$ $=\left\{(z, y): g(z)=p_{2}(\sigma(y))\right\}$. As in Proposition 5.3, $p_{Y}^{*} \eta^{\prime}$ is locally $L^{1}$ on $W$ and hence $p_{Z *} p_{Y}^{*} \eta^{\prime}$ is locally $L^{1}$ on $Z$. As before $p_{2} \circ \sigma: Y \rightarrow V$ is a product over $V-X$, so $g^{*} T_{\eta} i_{*}[\varphi]=p_{Z *} p_{Y}^{*} \eta^{\prime}$ almost everywhere.

(c) Continuity of fibering. We wish to generalize the fibering theorem of [19].

Proposition 5.5. Suppose that $W \subset V$ are complex manifolds, $d=$ codimension $W$, and suppose that a subvariety $X \subset V$ is endowed with a holomorphic map $\pi: X \rightarrow U$, $U$ a locally irreducible complex space, such that for each $X_{z}=\pi^{-1}(z), X_{z}$ has dimension $k$ and $X_{z} \cap W$ has dimension $k-d$. Then given any $\eta \in A^{p, q}(V\{W\})$ the current $\left[X_{z}\right] \wedge \eta$ varies continuously with $z$ in the current topology (also in the flat topology [8], [19]), where $\left[X_{z}\right]$ is the variety $X_{z}$ with the multiplicity of the map $\pi$ at $z$.

Remark. $\left[X_{z}\right]=\langle[X], \pi, z\rangle$ in the notation of [19].

Proof. We may assume that $d=1$ since if we set $X^{\prime}=\sigma^{-1}(X)$ for $\sigma: V[W]$ $\rightarrow V, W^{\prime}=E$ and $V^{\prime}=V[W], \pi^{\prime}=\pi \circ \sigma$ and $\eta^{\prime}=\sigma^{*} \eta$ we have a configuration that satisfies the hypotheses of the theorem. Since $\sigma_{*}\left(X_{z}^{\prime} \wedge \eta^{\prime}\right)=X_{2} \wedge \eta$ it will suffice to prove this case.

The currents $[X] \wedge \eta$ and $\left[X_{z}\right] \wedge \eta$ are defined by Proposition 5.3. If $\lambda$ is any smooth function which is $\equiv 1$ in a neighborhood of $W$, then $\left[X_{z}\right] \wedge(1-\lambda) \eta$ varies continuously with $z$ by the fibering theorem of [19]. We wish to show that given any $\varepsilon>0$ and any compact $K \subset V$ we can choose a $\lambda$ such that for any smooth form $\psi$ on $V$ supported on $K$,

$$
\left|\left[X_{\mathbf{z}}\right] \wedge \lambda \eta(\psi)\right|<\varepsilon\|\psi\|_{K}
$$

for all $z \in U$, where $\|\psi\|_{K}$ is the sup norm on $K$.

Since $d=1$, locally $\eta=t^{-1} d t \wedge \varphi_{1}+\varphi_{2}, \varphi_{i}$ smooth. Since the theorem is local we may assume $\eta=t^{-1} d t$ and then patch with a partition of unity.

Now let us resolve the singularities of $X$ by a proper map $\tau: \not{X} \rightarrow X$ so that $\tau^{-1}(X \cap W)=D$ is a divisor with normal crossings. As in the proof of Proposition 5.3, $D=\left\{z_{1} \cdots z_{k}=0\right\}$ in local coordinates and

$$
\tau^{*}\left(\frac{d t}{t}\right)=\sum_{i=1}^{k} n_{i} z_{i}^{-1} d z_{i}+C^{\infty} \text { term. }
$$

Since $\lim _{\delta \rightarrow 0}\left|\int_{|z|<\delta} z^{-1} d z \wedge d \bar{z}\right|=0$ in $\mathbf{C}$, we may find a neighborhood $N$ of $D \cap t^{-1}(K)$ such that (5.6) holds for any $0 \leq \lambda \leq 1$ such that supp $\lambda \circ \tau \subset N$.

(d) Generalizations of residue forms. Theorem 5.3 generalizes Lemma 1 of [18] which says that for any $g: X \rightarrow \mathbf{C}^{n}$ which is not identically zero the forms $g^{*}\left(\eta \wedge \omega^{r}\right)$ and $g^{*}\left(\omega^{r}\right)$ are locally $L^{1}$ on $X$, where 


$$
\eta=(2 \pi i)^{-1} d^{\prime} \log \left(\left|z_{1}\right|^{2}+\cdots+\left|z_{n}\right|^{2}\right)
$$

and $\omega=d^{\prime \prime} \eta$ are the forms of Example 1.4. In proving residue theorems it is frequently useful to prove that a given residue form is locally $L$, and such forms are of ten pull-backs by $g^{*}$ of simpler forms. For this reason it would be useful to extend the theorems of this section to a wider class of forms; we will indicate how this can be done.

Let $W$ be a submanifold of $V$; then a form $\eta, C^{\infty}$ on $V-W$, is said to have general residue type along $W$ if the form $\sigma^{*} \eta$ on $V[W]$ has singularities which are sums of the following forms near $E=\sigma^{-1}(w)$ : The local defining equation for $E$ is $t=0$.

(a) $\left(\log |t|^{2}\right)^{k} \psi$,

(b) $\left(\log |t|^{2}\right)^{k} t^{-1} d t \wedge \psi$,

(c) $\left(\log |t|^{2}\right)^{k} \bar{t}^{-1} d \bar{t} \wedge \psi$,

where $\psi$ is a form $C^{\infty}$ on $V[W]-E$ and bounded near each point of $E$.

Forms of this type share the property of $t^{-1} d t$ that $g^{*}$ of such a form is always locally $L^{1}$ on an irreducible $Z$ provided $g(Z) \oplus\{t=0\}$. The proof is the same as given in Proposition 5.3. Resolve the singularities of $Z$ to $\tau: Z \rightarrow Z$ such that $D=\{t \circ \tau=0\}$ is a divisor with normal crossings. Then $t \circ \tau=z_{1}^{n_{1}}, \ldots, z_{k}^{n_{k}}$ times a unit, $z_{i}$ being coordinates. It is trivial that for such $t \circ \tau$ the singularities (a), (b), (c) are locally $L^{1}$ on $Z$ and we apply $\tau_{*}$ to get Proposition 5.3 for these forms. Thus we claim the following proposition is true.

Proposition 5.7. Propositions 5.2, 5.3, and 5.5 are true for forms $\eta$ of general residue type.

Proof. Same as for Propositions 5.2, 5.3, and 5.5.

Observe that singularities such as $|t|^{-1} d t$ are included in these forms since this is $\left(t|t|^{-1}\right) t^{-1} d t$ and $t|t|^{-1}$ is bounded.

These forms are not closed under exterior multiplication since $|t|^{-2} d t \wedge d \bar{t}$ is not $L^{1}$. However, for such a form $\eta, \sigma_{*} \eta \wedge \varphi$ is locally $L^{1}$ on $V$ if the degree of the form $\eta<2$ codim $W$ and degree $\sigma_{*} \eta \wedge \varphi=2 \operatorname{dim} V$, since for any coordinate $w_{i}$ on $V$ constant on $W, \sigma^{*} d w_{i}=t d \zeta_{i}+\zeta d t$. The $d t$ term is killed by $d t \wedge d \bar{t}$ and $t d \zeta_{i} \wedge|t|^{-2} d t \wedge d \bar{t}=d \zeta_{i} \wedge \bar{t}^{-1} d t \wedge d \bar{t}$ which is $L$. Since degree $\eta$ $<2 \operatorname{codim} W$, any form $\varphi$ of complementary degree must involve such a coordinate $w_{i}$. Thus $\sigma_{*} \eta$ is also locally $L$.

Proposition 5.3 is not true in general for forms $\eta$ with the singularity of $\sigma^{*} \eta$ being $|t|^{-2} d t \wedge d \bar{t}$, but if $g^{-1}(W)$ is a submanifold of a manifold $Z$ with codimension $d$, where $d$ also is the codimension of $W$ in $V$, then Proposition 5.3 does hold if $\operatorname{deg} \eta<2 d$.

For example we have the following:

Proposition 5.8. Let $g:\left(g_{1}, \ldots, g_{n}\right): U \rightarrow C^{n}$ be a holomorphic map on $0 \in U$ $\subset \mathbf{C}^{n}$ such that $\mathrm{g}^{-1}(0)=0$. Then the form 


$$
(\log |g|)^{k}|g|^{-|K|-|L|} d g^{K} \wedge d \bar{g}^{L}
$$

is locally $L^{1}$ if $|K|+|L|<2 n$, where $d g^{K}=d g_{k_{1}} \wedge \cdots \wedge d g_{k_{r}} r=|K|$, etc.

Proof. The proof is indicated above since this form is $g^{*} \eta, \eta=(\log |z|)^{k}$ $|z|^{-|K|-|L|} d z^{K} \wedge d \bar{z}^{L}$. The worst singularities of $\sigma^{*} \eta$ on $C^{n}$ are $|t|^{-2} d t \wedge d \bar{t}$ which is cancelled by the zeroes of a $2 n-|K|-|L|$ form $\varphi$ pulled up to the fiber product. A complete proof of this is given in Lemma 5.6 of [5], along the lines indicated here; this was previously proved in [30].

A final remark is that if one wishes to admit more general currents than $L^{1}$ forms, one can look at the principal value of $d f / f^{n}$ for $n>1$. This theory has been developed in [14]. One could also look at currents $\sigma_{*} u$, where $u$ is a current on $V[W]$ of this type and could probably establish some of the results of this paper for these currents.

6. Intersection of complex subvarieties. Given two complex subvarieties $X, Y$ $C V$, a complex manifold of dimension $n$, if $\operatorname{dim}(X \cap Y)=\operatorname{dim} X+\operatorname{dim} Y$ $-n$, the intersection $X \cdot Y$ is defined. This $X \cdot Y$ consists of the subvariety $X \cap Y$ with a certain multiplicity assigned to each component. In [3], [7], and [19], $X \cdot Y$ is defined by homology, local analytic geometry, and measure theory, respectively; in the latter it is also proved that the definitions coincide.

In this section we will define $X \cdot Y$ by means of residues. This was already done in [18] for a special residue form constructed from the Bochner-Martinelli kernel; but we generalize this result to the residue forms of $\$ 83$ and 4 .

We will first deal with the case of a subvariety intersecting a submanifold transversally. Then using local perturbations of $X$ and $Y$ and the continuity proposition 5.5 we will show that our intersection definition coincides with that of [19].

Lemma 6.1. Let $X$ be a k-dimensional complex subvariety of the complex $n$ manifold $V$ and let $W$ be an m-dimensional complex submanifold of $V$ such that $\operatorname{dim}(X \cap W)=k+m-n$. If the intersection of $\operatorname{Reg} X$ and $W$ is transversal and if $\operatorname{dim}(\operatorname{Sing} X \cap W)<k+m-n$, then for any $\eta \in A^{r}(V\{W\})$ with

$$
d[\eta]-[d \eta]=i_{*}[\varphi] \text { for } \varphi \in A^{s}(W)
$$

and $i: W \rightarrow V$ the inclusion, we have the following equation of currents:

$$
d([X] \wedge \eta)-[X] \wedge d \eta=i_{*}([X \cap W] \wedge \varphi) .
$$

Remark. By hypothesis $[X \cap W]=[X \cdot W]$ since the multiplicity of a transversal intersection is one.

Proof. We first observe that $[X] \wedge \eta=\sigma_{*}\left(\left[X^{\prime}\right] \wedge \sigma^{*} \eta\right),[X] \wedge d \eta=\sigma_{*}\left(\left[X^{\prime}\right]\right.$ $\left.\wedge d \sigma^{*} \eta\right)$, where $X^{\prime}=\sigma^{-1}(X) \subset V[W]$. This is true since for any smooth form $\alpha,[X] \wedge \eta(\alpha)=\int_{\operatorname{Reg} X} \eta \wedge \alpha=\int_{\operatorname{Reg} X-W} \eta \wedge \alpha=\int_{\operatorname{Reg} X^{\prime}-E} \sigma^{*} \eta \wedge \sigma^{*} \alpha$ since $X$ 
$\cap W$ has measure zero in $X$ by hypothesis. We observe that the integrals converge by Proposition 5.3.

Now since these are equations of currents we can localize, or equivalently, we break up $\sigma^{*} \eta$ by a partition of unity so that we may assume $\sigma^{*} \eta=(2 \pi i t)^{-1} d t$ $\wedge \psi+\zeta$, where $t=0$ is a local defining equation for $E=\sigma^{-1}(W)$ and $\psi$ and $\zeta$ are smooth forms supported in the domain of $t$. Of course $\eta=\sigma_{*} \sigma^{*} \eta$. For this $\eta$

$$
u=d\left(\left[X^{\prime}\right] \wedge \sigma^{*} \eta\right)-\left[X^{\prime}\right] \wedge d \sigma^{*} \eta=\left(d\left(\left[X^{\prime}\right] \wedge\left(2 \pi i t^{-1}\right) d t\right)\right) \wedge \psi
$$

Let $U$ be an open set contained in domain $t$. The current

$$
v=d\left(\left[X^{\prime}\right] \wedge(2 \pi i t)^{-1} d t\right)
$$

clearly does not depend on the choice of $t$. We claim that the restriction $\left.v\right|_{U}=\left[X^{\prime} \cap E \cap U\right]$. If this is verified, then we will have, by (6.2), $u$ $=\left[X^{\prime} \cap E\right] \wedge \psi$.

We wish to show that $\left.v\right|_{U}$ is determined by its restriction to $U-$ $\operatorname{Sing}\left(X^{\prime} \cap E\right)$. One way to prove this is to follow the proof of Proposition 5.3 and lift the form $(2 \pi i t)^{-1} d t$ by $\tau=X \rightarrow X^{\prime}$, a resolution of the singularities of $X$ such that $\{t \circ \tau=0\}$ is a divisor with normal crossings $D$, counted with multiplicities. Then $d\left[\tau^{*}(2 \pi i t)^{-1} d t\right]=[D]$ and $\tau_{*}[D]=d\left[(2 \pi i t)^{-1} d t\right]$ on $X^{\prime}$ and this is the current defined by a locally $L^{1}$ function on $X^{\prime} \cap E$ by Proposition 5.1. Since this is a closed current the function is constant on $\operatorname{Reg}\left(X^{\prime} \cap E\right)$. Since $d t \neq 0$ for the set $\operatorname{Reg} X^{\prime} \cap E$ dense in $\operatorname{Reg}\left(X^{\prime} \cap E\right)$, by an easy computation, essentially given in Example 1.2, this $L^{1}$ function $=1$.

A second way of concluding this is by a general theorem of Federer on the supports of flat currents, of which $v$ is an example (cf. [8] or discussion in [19] about measure support).

We have now verified that

$$
d([X] \wedge \eta)-[X] \wedge d \eta=\sigma_{*} u=\sigma_{*}\left(\left[X^{\prime} \cap E\right] \wedge \psi\right) .
$$

Now for $X=V$, this says that $\sigma_{*}([E] \wedge \psi)=\varphi$. That is the integration of $\psi$ over the fiber of the $\mathrm{P}^{m-1}$ bundle $E$ over $W$ is $\varphi$. But $X^{\prime} \cap E$ is just the same bundle restricted to a $\mathbf{P}^{m-1}$ bundle over $X \cap W$. Thus $\sigma_{*}\left(\left[X^{\prime} \cap E\right] \wedge \psi\right)=[X \cap W]$ $\wedge \varphi$.

Theorem 6.3. Let $X$ be a $k$-dimensional complex subvariety of the complex $n$ manifold $V$ and let $W$ be an m-dimensional complex submanifold of $V$ such that $\operatorname{dim}(X \cap W)=k+m-n$. For any $\eta \in A^{r}(V\{W\})$ with

$$
d[\eta]-[d \eta]=i_{*}[\varphi] \text { for } \varphi \in A^{s}(W)
$$

and $i: W \rightarrow V$ the inclusion, we have the following equation of currents:

$$
d([X] \wedge \eta)-[X] \wedge d \eta=i_{*}([X \cdot W] \wedge \varphi) .
$$

Corollary 6.4. For $X$ and $V$ as in Theorem 6.3, let $j: Y \rightarrow V$ be a subvariety of dimension $m$ and let $\mu \in A^{2 n-1}(V \times V\{\Delta\})$ be such that $d[\mu]-[d \mu]=[\Delta]$ and 
$p_{i}:$ supp $\mu \rightarrow V$ is proper, $i=1,2$. Then for any $\psi \in A^{s}(Y)$, if $[\rho]=T_{\eta} j_{*}[\psi](c f$. Proposition 4.7(c)) then $d([X] \wedge \rho)-[X] \wedge d \rho=[X \cdot Y] \wedge \psi$.

Proof of Corollary 6.4. The current $[X] \wedge \rho=p_{2 *}\left([X \times Y]^{\wedge} \wedge \mu \wedge p_{1}^{*} \psi\right)$; the proof is implicit in Proposition 5.4, although this case is much simpler. If in Theorem 6.3 we replace $V$ by $V \times V, W$ by $\Delta, X$ by $X \times Y$ and $\eta$ by $\mu \wedge p_{1}^{*} \psi$ we are done, using the fact that $p_{2 *}[X \times Y \cdot \Delta]=[X \cdot Y][19$, part 4.1].

Corollary 6.5. The results in Theorem 6.3 and Corollary 6.4 remain true if there is a form $\rho$ with $d^{\prime \prime}[\rho]-\left[d^{\prime \prime} \rho\right]=i_{*}[\varphi]$ or $[\Delta]$, respectively. Then $d^{\prime \prime}([X] \wedge \rho)$ $-[X] \wedge d^{\prime \prime} \rho=[X \cdot W] \wedge \varphi$, etc.

Proof of Corollary 6.5. Observe that $d[\rho]-[d \rho]=d^{\prime \prime}[\rho]-\left[d^{\prime \prime} \rho\right]$ since

$$
d^{\prime}\left[t^{-1} d t \wedge \psi\right]=\left[t^{-1} d t \wedge d^{\prime} \psi\right]
$$

Proof of Theorem 6.3. Since the theorem is local we may assume that $V$ is an open subset of $\mathbf{C}^{n}$.

We wish to look at the family of all translations of $X$. Let $Z \subset X \times \mathbf{C}^{n}$ be $\{(x, z): x-z \in X\}$ and $\pi: Z \rightarrow C^{n}$ be the projection. Now for any open subset $U \subset V$ with compact closure. $\bar{U} \subset V$ there is a polydisk $0 \in \Delta \subset \mathbf{C}^{n}$ such that $\pi^{-1}(\Delta) \cap Z \cap U \times C^{n}$ is a subvariety of $U \times C^{n}$. (We are taking translates of $X$ and thus translating boundary points of $X$ into the interior of $V$; for any relatively compact $U$, small translations of $X$ are still subvarieties of $U$.) Call this subvariety $Z^{\prime}$ and let $W^{\prime}=(W \cap U) \times \Delta$ and $\eta^{\prime}=p^{*} \eta$, where $p=V \times C^{n}$ $\rightarrow V$. After possibly restricting $\Delta$ further, each $Z_{z}^{\prime}=X+z$ intersects $W^{\prime}$ in dimension $k+m-n$.

Thus $\pi: Z^{\prime} \rightarrow \Delta$ and $W^{\prime} \subset U \times \Delta$ satisfy the hypotheses of Proposition 5.5. This says that the currents $\left[Z_{z}^{\prime}\right] \wedge \eta^{\prime}$ and $\left[Z_{z}^{\prime}\right] \wedge d \eta^{\prime}$ vary continuously in $z$. Now if we set $X_{z}=X+z$, the translation of $X$ by $z$, then $\left[Z_{z}^{\prime}\right]=\left[X_{z} \times\{z\}\right]$. The multiplicities are one because the map $\pi: Z^{\prime} \rightarrow \Delta$ is a local product. Thus the current $d\left(\left[X_{z}\right] \wedge \eta\right)-\left[X_{z}\right] \wedge d \eta=p_{*}\left(d\left(\left[Z_{z}^{\prime}\right] \wedge \eta^{\prime}\right)-\left[Z_{z}^{\prime}\right] \wedge d \eta\right)$, which is defined on $U$, varies continuously in $z$.

Now we claim that for almost all $z$ in $\Delta, X_{z}$ satisfies the hypothesis of Lemma 6.1. Suppose that the coordinates in $V$ have been chosen initially so that $W=L^{-1}(0)$ for some linear map $\mathbf{C}^{n} \rightarrow \mathbf{C}^{n-m}$. If

$$
B=\left\{x \in \operatorname{Reg} X: \operatorname{rank} d\left(\left.L\right|_{X}\right)_{x}<n-m\right\},
$$

by Sard's theorem $L(B)$ has measure 0 . But $L(X)$ contains a neighborhood of 0 since $\operatorname{dim}\left(L^{-1}(w) \cap X\right)=k+m-n$ for small $w$ by upper semicontinuity of dimension [12, p. 159]. Also the set of $w$ such that $\operatorname{dim}\left(L^{-1}(w) \cap \operatorname{Sing} X\right)=k+m-n$ is a set of measure zero also by Sard's theorem.

Thus we have arrived at the following situation. We have two continuous families of currents depending on $z \in \Delta$ :

(a) $d\left(\left[X_{z}\right] \wedge \eta\right)-\left[X_{z}\right] \wedge d \eta$,

(b) $i_{*}\left(\left[X_{z} \cdot W\right] \wedge \varphi\right)$, which is continuous by [19, Definition 4.1.3 and Theorem 3.3.2].

Furthermore, the families (a) and (b) are equal on a dense subset of $\Delta$ by 
Lemma 6.1 and the remarks above; consequently they are equal for all $z$. If we set $z=0$ we are done.

Corollary 6.6. If $X$ and $Y$ are $r$ - and s-subvarieties of $V$ such that $X \cdot Y$ is defined and $\varphi$ and $\psi$ are forms cohomologous to $X$ and $Y$ in $H^{*}\left(V, \Omega^{*}\right) \simeq H^{*}\left(\Gamma K_{V}^{*}\right)$ or $H^{*}\left(V, \hat{\Omega}^{*}\right) \simeq H^{*}\left(\Gamma F^{*} \mathcal{K}_{V}^{*+*}\right)$ then $[X \cdot Y]$ is cohomologous to $\varphi \wedge \psi$ in the respective group.

Proof. By Theorem 4.11 there is an $\eta \in A^{n, n}(V \times V[\Delta])$ such that $[Y]$ $=d^{\prime \prime} T_{\eta}[Y]+\psi_{1}$, where $\psi_{1}$ is a smooth form (if we are treating the case of Dolbeault cohomology) and $\psi_{1}-\psi=d^{\prime \prime} \alpha$, for some smooth $\alpha$. By Corollary 6.5, $[X \cdot Y]=d^{\prime \prime}\left([X] \wedge T_{\eta}[Y]\right)+[X] \wedge \psi_{1}=[X] \wedge \psi+d^{\prime \prime}\left([X] \wedge\left(T_{\eta}[Y]+\alpha\right)\right)$. Thus $[X \cdot Y]$ is cohomologous to $[X] \wedge \psi$. But $[X]-\varphi=d^{\prime \prime} u$ for some current $u$ of type $(u-k, n-k-1)$. Thus $[X \cdot Y]$ is cohomologous to $\varphi \wedge \psi+$ $d^{\prime \prime}(u \wedge \psi)$. The argument for the cohomology of $\hat{\Omega}$ is the same.

Remark. If we define the residue $\operatorname{Res}_{W} \eta=d[\eta]-[d \eta]$, this says that the residue of the restriction to $X$ is the intersection of $X$ with the residue; this idea is developed more fully in the next section.

The local residue theory of this section can be used to study the Lefschetz fixed point theorem and the theory of Bott and Baum [1]. We hope to treat this in another place.

7. An intersection formula. (a) Let $W$ be a $k$-dimensional complex submanifold of the complex $n$-manifold $V$ and let $\varphi$ be a form in $F^{j} A_{c}^{r}(W)$ such that $d \varphi=0$. Then by Theorem 2.13(a) there is a $\psi \in F^{j+n-k} A_{c}^{r+2 n-2 k}(V)$ and an $\eta$ $\in F^{j+n-k} A_{c}^{r+2 n-2 k-1}(V\{W\})$ such that $i_{*}[\varphi]=d[\eta]+[\psi]$, where $i: W \rightarrow V$ is the inclusion.

If $[X]$ is the current defined by an $s$-dimensional complex subvariety of $V$ with both positive and negative integer multiplicities, then if $[X]$ is homologous to zero (to be made precise below) we will show that there exists a Z-chain $C$, a current, such that $d C=[X]$ and

$$
C(\psi)=(C \cdot W)(\varphi)
$$

for any closed form $\varphi \in F^{s+k-n+1} A_{c}^{2(s+k-n)+1}(W)$ as above. The intersection chain $W \cdot C$ will be defined below so that $d(W \cdot C)=W \cdot[X]$ as defined in the previous section.

This formula can be applied to establish certain functorial properties of the Abel-Jacobi mappings of complex analytic cycles on compact Kähler manifolds into the associated intermediate Jacobian varieties. This argument is indicated and verified in part in [10]; a complete discussion will appear in [20].

To explain the nature of (7.1) a bit further, if $C$ is a differentiable singular chain, the equations becomes

$$
\int_{C} \psi=\int_{C \cdot W} \varphi
$$


The analogous situation on a real manifold $M$ occurs if $d C=0$. Say, for simplicity, that $\operatorname{dim} C+\operatorname{dim} V=\operatorname{dim} M$ and $\varphi=1$, the constant function. Then $W=d\left[\eta_{W}\right]+\psi_{W}$ and $C=d\left[\eta_{C}\right]+\psi_{C}$, where $\psi_{W}$ and $\psi_{C}$ are $C^{\infty}$. Equation (7.2) becomes $\int_{W \cdot C} 1=\int_{C} \psi_{W}=\int_{M} \psi_{C} \wedge \psi_{M}$ since $d \eta_{C}\left(\psi_{M}\right)=0$. In other words this says that intersection in homology is dual to exterior product in de Rham theory; this can be proved using smoothing [28]. To show (7.2) in the real case it is essential to assume that $d C=0$ since the difference in the two sides should be $d C\left(\eta_{W}\right)$ if this is defined. In the complex case we can extend this result by making restrictions on the type of $d C$ and $\varphi$ which make this term vanish.

First we wish to define an integer homology theory with a complex of chains large enough to include all complex analytic cycles $[X]$. We designate by $I_{r}^{\text {loc }}(V) \subset \mathscr{D}_{r}^{\prime}(V)$ the group of locally integral currents in the sense of Federer and Fleming [8]. The elements of this group are chains which have tangent spaces almost everywhere and whose boundaries are chains of the same sort; thus $d\left(I_{r}^{\text {loc }}(V)\right) \subset I_{r-1}^{\text {loc }}(V)$. For any $s$-dimensional complex subvariety $X \subset V,[X]$ $\in I_{2 s}^{\text {loc }}(V)$ since $[X]$ is given by integration over the submanifold $\operatorname{Reg} X$ with locally finite volume in $V$ and since $d[X]=0$.

We will need to know the support property: if $u \in I_{r}^{\text {loc }}(V)$ and supp $u$ is contained in a submanifold $i: W \rightarrow W$, then $u=i_{*} u^{\prime}$ for a unique $u^{\prime} \in I_{r}^{\text {loc }}(W)$. Thus if $W$ has dimension less than $r, u=0$ [8, pp. 372-373]. Also, the integral currents $I_{r}(V)=I_{r}^{\mathrm{loc}}(V) \wedge \delta_{r}^{\prime}(V)$ are the chains with compact support; if $f: V_{1} \rightarrow V_{2}$ is smooth, $f_{*} u \in I_{r}\left(V_{2}\right)$ whenever $u \in I_{r}\left(V_{1}\right)$.

Also, if $u_{i} \in \mathscr{D}_{n}^{\prime}\left(V_{i}\right), i=1,2$, the product $u_{1} \times u_{2} \in \mathscr{D}_{n+n_{2}}^{\prime}\left(V_{1} \times V_{2}\right)$ is the unique current such that $u_{1} \times u_{2}\left(p_{1}^{*} \varphi_{1} \wedge p_{2}^{*} \varphi_{2}\right)=u_{1}\left(\varphi_{1}\right) u_{2}\left(\varphi_{2}\right)$ for $\varphi_{i} \in A_{c}^{s_{i}}\left(V_{i}\right)$ and the projections $p_{i}$. If $u_{i} \in I_{n}^{\text {loc }}\left(V_{i}\right)$, then $u_{1} \times u_{2} \in I_{n+u_{2}}^{\text {loc }}\left(V_{1} \times V_{2}\right)$ and $d\left(u_{1} \times u_{2}\right)= \pm d u_{1} \times u_{2} \pm u_{1} \times d u_{2}$.

Suppose $f:[0,1] \times V \rightarrow V$ is a smooth map, where $[0,1] \subset \mathbf{R}$ is the closed unit interval. We shall also designate by $[0,1]$ the current in $I_{1}(R)$ given by integrating a 1-form over the unit interval. Then for any current $u \in \mathcal{E}_{r}^{\prime}(V)$, we will define the homotopy operator $K_{f}: \varepsilon_{r}^{\prime}(V) \rightarrow \varepsilon_{r+1}^{\prime}(V)$ by $K_{f}(u)=f_{*}([0,1] \times u)$. Then for any such $u$,

$$
d K(u)-K(d u)=f_{1 *} u-f_{0 *} u,
$$

where $f_{i}: V \rightarrow V$ means $f_{i}(x)=f(i, x)$. Observe that if $u \in I_{r}(V)$, all the currents in this equation are integral currents (cf. $[8$, p. 363]).

For the rest of this section, we will, after some preparation, define an intersection $u \cdot W$ for certain currents $u$. This definition will be made so that (7.1) holds for such currents. Then, since for once we are interested in homology with $\mathbf{Z}$ coefficients, we will define a subgroup $I_{r}^{\text {loc }}(V\{W\})$ of integral currents which have intersections with $W$ and show that $H_{*}\left(I^{\text {loc }}(V\{W\})\right) \simeq H_{*}(V, \mathbf{Z})$. This will demonstrate the existence of an integral chain $C$ such that (7.1) holds.

(b) Extensions and residues of currents along $W$. If $u \in \mathscr{D}_{r}^{\prime}(V-W)$ is a current 
of order zero, then it may be possible to extend $u$ to a current $\tilde{u} \in \mathscr{D}_{r}^{\prime}(V)$. Since the coefficients of $u$ are measures, it is possible that for any $\varphi \in A_{c}^{r}(V)$ the restriction $\left.\varphi\right|_{V-W}$ is integrable with respect to $u$; in this case we set $\tilde{u}(\varphi)$ $=u\left(\left.\varphi\right|_{V-W}\right)$ (see [23] for details). If $\tilde{u}$ exists we call it the simple extension of $u$. If $0 \leq \lambda_{i} \leq 1$ is a sequence of smooth functions in $V-W$ such that supp $\lambda_{i}$ $\cap \operatorname{supp} \varphi \cap V-W$ is compact for all $i$ and if $\lambda_{i}(x) \rightarrow 1$ for all $x \in V-W$ as $i \rightarrow \infty$, then $\tilde{u}(\varphi)=\lim _{i \rightarrow \infty} u\left(\lambda_{i} \varphi\right)$ if $\tilde{u}$ exists.

Now if $u \in \mathscr{D}_{r}^{\prime}(V-W)$ then $\sigma_{*}^{-1} u \in \mathscr{D}_{r}^{\prime}(V[W]-E)$ is defined, since $\sigma$ : $V[W]-E \rightarrow V-W$ is biholomorphic.

Definition 7.3. If $v \in \mathscr{D}_{r}^{\prime}(V)$ is of order zero, then the simple lift $v_{0}$ $=\left(\sigma_{*}^{-1}\left(\left.v\right|_{V-W}\right)\right)^{\sim} \in \mathscr{D}_{r}^{\prime}(V[W])$. If $v_{\sigma}$ exists, we say $v$ is simply liftable at $W$.

Notice that $\sigma_{*} v_{\sigma}=\left(\left.v\right|_{V-W}\right)^{\sim}$, which does not necessarily equal $v$ at points of $W$.

Definition 7.4. A current $u \in \mathscr{D}_{r}^{\prime}(V)$ is current transversal to $W$ if $u=\sigma_{*} u_{\sigma}$ and $d u=\sigma_{*}(d u)_{\sigma}$ and if $u \wedge \eta$ and $d u \wedge \eta$ are simply liftable at $W$ for all $\eta \in A^{s}(V\{W\})$, any $s$.

Remark. If $u$ and $d u$ are the currents of integration over manifolds or subvarieties $M$ and $\partial M$, then $u$ is current transversal if $M \cap W$ and $\partial M \cap W$ are sets of measure zero in $M$ and $\partial M$ and if $i^{*} \eta$ is locally $L^{1}$ on both $M$ and $\partial M$ ( $i$ being the inclusion). If the real dimension of $M$ is 0 or 1 , this means $M \cap W=\varnothing\left(\right.$ let $\left.\eta=t^{-1} d t\right)$. If $u=[X], X$ a complex subvariety, it suffices by Proposition 5.3 that no component of $X$ is contained in $W$. If $u=[\mu]$, $\mu \in A^{j}(V\{W\})$ is such that $d[\mu]=[d \mu]$, then $u$ is current transversal to $W$.

Definition 7.5. If $u \in \mathscr{D}_{r}^{\prime}(V)$ and $u$ and $d u$ are of order zero, then the residue

$$
\operatorname{Res}_{W} u=d u-\left(\left.(d u)\right|_{v-W}\right)^{\sim} .
$$

For this section we will write $\operatorname{Res} u=\operatorname{Res}_{W} u$.

The restriction above is merely defined by restricting measures. The equation $i_{*}[\varphi]=d[\eta]+[\psi]$ which we have encountered before in Theorem 2.13 may be written now as $i_{*}[\varphi]=\operatorname{Res}[\eta]$.

An interesting fact that follows from the support property proved by Federer is that there is a unique current res $u \in \mathscr{D}_{r-1}^{\prime}(W)$ such that $i_{*}$ res $u=\operatorname{Res} u$, if $i: W \rightarrow V$ is the inclusion. It is clear that $\operatorname{res}[\eta]=[\varphi]$ in the previous paragraph; indeed for any $\eta \in A^{s}(V\{W\})$, res $[\eta]$ is a smooth form.

(c) Intersections of currents with $W$.

Definition 7.6. A current $u \in \mathscr{D}_{r}^{\prime}(V)$ is of intersection type along $W$ if $u$ is current transversal to $W$ and if there is a (necessarily unique) current $u \cdot W$ $\in \mathscr{D}_{s}^{\prime}(W)$ such that $\operatorname{res}(u \wedge \eta)=(-1)^{s}(u \cdot W) \wedge$ res $\eta$ for all $\eta \in A^{q}(V\{W\})$, where $s=r-2 \operatorname{codim} W$.

Note. The sign is chosen so that $d(u \cdot W)=d u \cdot W$ if both are defined.

We observe from the definition that if $\eta$ is any form in $A^{2 d-1}(V\{W\})$ such that $\operatorname{Res}[\eta]=[W]$, then $u \cdot W=\operatorname{res}(u \wedge \eta)$. In particular, if $d=\operatorname{codim}_{\mathbf{C}} W=1$, $u \cdot W$ is the current locally given by $(-1)^{s} \operatorname{res}\left(u \wedge t^{-1} d t\right)$ where $W=\{t=0\}$ 
locally. Since res $\left(u \wedge t^{-1} d t\right)$ is independent of the choice of $t$, any $u$ which is current transversal to $W$, with $d=1$, is of intersection type.

Remark. It may be worthwhile to point out that if $N_{\varepsilon}$ is the $\varepsilon$-tubular neighborhood about $W$ and $\chi_{e}$ is the characteristic function of $N_{e}$, we may define

$$
\text { “v } \partial N_{\varepsilon} "= \pm\left(d\left(v \wedge\left(1-\chi_{e}\right)\right)-(d v) \wedge\left(1-\chi_{e}\right)\right) .
$$

Then $v \cdot W=\lim _{e \rightarrow 0}$ “. $\partial N_{e} " \wedge \eta$, if $\operatorname{Res} \eta=[W]$. The intersection of $v$ with $W$ is the limit of the integration of $\eta$ over the intersection of $v$ with the normal sphere bundle.

Definition 7.7. $I_{r}^{\mathrm{loc}}(V\{W\})$ is the group of currents $u \in I_{r}^{\mathrm{loc}}(V)$ such that $u$ and $d u$ are of intersection type along $W$ and $u \cdot W \in I_{r-2 d} \operatorname{loc}_{d}(W), d=\operatorname{codim} W$. We also require that supp $u \cap W=\varnothing$ if $r<2 d$.

Notice that $(d u) \cdot W=d(u \cdot W)$ is also a locally integral current.

Proposition 7.8. If $X$ is a complex $k$-subvariety of $V$ such that $X \cdot W$ is defined as in [19], then $[X] \in I_{2 k}^{\text {loc }}(V\{W\})$ and $[X] \cdot W=[X \cdot W]$.

Proof. This is a corollary of Theorem 6.3.

A reason that $[X]$ satisfies Definition 7.7 is that $[X]_{\sigma} \cdot E$ is locally the product current of $[X] \cdot W$ and the fiber $\mathbf{P}^{d-1}$ of the bundle $\pi: E \rightarrow W$ induced by $\sigma$. An invariant way of expressing that a current $v \in \mathscr{D}_{r}^{\prime}(E)$ is locally a product with the fiber in this way is that there exists a current $v^{\prime} \in \mathscr{D}_{r-2 d+2}^{\prime}(W)$ such that $v(\psi)=v^{\prime}\left(\pi_{*} \psi\right)$ for any $\psi \in A_{c}^{r}(E)$. The smooth form $\pi_{*} \psi$ is the result of integrating along the fiber, i.e. $\left[\pi_{*} \psi\right]=\pi_{*}[\psi]$. In this case we say that $v=\pi^{*} v^{\prime}$ since this map extends the map $\pi^{*}$ on forms. This local product criterion actually characterizes currents of intersection type.

Proposition 7.9. $A$ current $v \in \mathscr{D}_{k}^{\prime}(V)$ which is current transversal to $W$ is of intersection type if and only if there exists a current $v^{\prime} \in \mathscr{D}_{k-2 d}^{\prime}(W)$ such that $\pi^{*} v^{\prime}=v_{0} \cdot E$; this $v^{\prime}$ is necessarily $v \cdot W$. Here $\pi: E \rightarrow W$ as above and codim $W$ $=d$.

Proof. For $v$ of intersection type and for any $\eta \in A^{s}(V\{W\}), \operatorname{Res}(v \wedge \eta)$ $=\sigma_{*} \operatorname{Res}\left(v_{\sigma} \wedge \sigma^{*} \eta\right)$, which means by Definition 7.7 that

$$
v \cdot W \wedge \operatorname{res}[\eta]=\pi_{*}\left(v_{\sigma} \cdot E \wedge \operatorname{res}\left[\sigma^{*} \eta\right]\right) .
$$

Given any $\psi \in A_{c}^{r-2}(E)$ we can find by partition of unity an

$$
\eta^{\prime} \in A_{c}^{r-1}(V[W]\{E\})
$$

such that $\operatorname{res}\left[\eta^{\prime}\right]=[\psi]$. Let $\eta=\sigma_{*} \eta^{\prime} ;$ then $\sigma^{*} \eta=\eta^{\prime}$ and $\operatorname{Res}[\eta]=\sigma_{*} \operatorname{Res}\left[\sigma^{*} \eta\right]$ implies that $\operatorname{res}[\eta]=\left[\pi_{*} \psi\right]$. Substituting this into (7.10) gives $v \cdot W\left(\pi_{*} \psi\right)=v_{0}$ - $E(\psi)$, which proves half the proposition.

It is straightforward to reverse the argument: $\operatorname{Res}(v \wedge \eta)=\sigma_{*} \operatorname{Res}\left(v_{\sigma} \wedge \sigma^{*} \eta\right)$ implies $\operatorname{res}(v \wedge \eta)=(-1)^{k} \pi_{*}\left(v_{0} \cdot E \wedge \operatorname{res}\left[\sigma^{*} \eta\right]\right)=(-1)^{k} v^{\prime} \wedge \pi_{*} \operatorname{res}\left[\sigma^{*} \eta\right]=v^{\prime}$ $\wedge$ res $[\eta]$ by hypothesis, since $\pi_{*}\left(\pi^{*} u \wedge \psi\right)=u \wedge \pi_{*} \psi$. This last is true since for any $\varphi$ of the right degree $\pi_{*}\left(\pi^{*} u \wedge \psi\right)(\varphi)=\pi^{*} u\left(\psi \wedge \pi^{*} \varphi\right)=u\left(\pi_{*}\left(\psi \wedge \pi^{*} \varphi\right)\right)$ by definition; but $\pi_{*}\left(\psi \wedge \pi^{*} \varphi\right)=\left(\pi_{*} \psi\right) \wedge \pi^{*} \varphi$ (check on another test form). 
This criterion lets us say that any real semianalytic chain which is current transversal to $W$ has intersection type. It also makes clear that oriented submanifolds intersecting $W$ transversally have intersection type as currents and that the intersections are the same.

(d) Homology theory. In this section we will prove that $H_{*}\left(I^{\mathrm{loc}}(V\{W\})\right)$ is the usual homology of $V$ with coefficients in $\mathbf{Z}$. The homology theory that we will use is Borel-Moore homology, which agrees with singular homology on manifolds.

Given a family of supports $\Phi$, let $I_{r}^{\Phi}(V\{W\})$ be the group of currents $u \in I_{r}^{\text {loc }}(V\{W\})$ such that supp $u \in \Phi$. Of course if $c=\{K \subset V ; K$ compact $\}$, $I_{r}(V\{W\})=I_{r}^{c}(V\{W\})$. These groups form a chain complex with boundary operator $d: I_{r}^{\Phi}(V\{W\}) \rightarrow I_{r-1}^{\Phi}(V\{W\})$ being the $d$ operator on currents. This differs by a sign of $(-1)^{r+1}$ from the usual boundary on chains, but we will use it for convenience.

Theorem 7.11. If $W$ is a complex submanifold of $V$ with $\operatorname{dim} W<\operatorname{dim} V$, then $H_{*}\left(I^{\Phi}(V\{W\})\right) \simeq H_{*}^{\Phi}(V, Z)$, the Borel-Moore homology of $V$ with integer coefficients.

Proof. By [4, p. 226], it suffices to prove the lemma below.

Lemma 7.12. Every $x \in V$ has a fundamental system of coordinate polydisk neighborhoods $\Delta_{i}$ such that $H_{r}\left(I .\left(\Delta_{i}\left\{\Delta_{i} \cap W\right\}\right)\right) \simeq H_{r}^{c}\left(\Delta_{i}, \mathbf{Z}\right) \simeq \mathbf{Z}$ if $r=0$ and $\simeq 0$ if $r \neq 0$.

Proof of lemma. First we check the case that $W=\varnothing$. Then if $\Delta_{i}$ is carried by the coordinate map onto a polydisk or any convex region in $\mathbf{C}^{n}$ containing $\mathbf{0}$, we let $f(t, x)=f(t x)$ give a map $f:[0,1] \times \Delta_{i} \rightarrow \Delta_{i}$. If $K_{f}$ is the associated homotopy, as described in $\S 7(\mathrm{a})$, then for any $u \in I_{r}\left(\Delta_{i}\right)$ with $d u=0, u=f_{0 *} u-d K_{f} u$. But $f_{0 *} u$ is an integral current supported on the 0 -dimensional manifold ( 0$)$, so by the support property of $\S 7(\mathrm{a}), f_{0 *} u=0$ if $r>0$ and $f_{0 *} u=n[0]$ if $r=0, n \in \mathbf{Z}$.

This proves the lemma and Theorem 7.9 if $W=\varnothing$ and we note here that the same proof and result hold if $V$ is just a $C^{\infty}$ real manifold. We will use this below.

Now we must prove (7.10) for $x \in W \neq \varnothing$. Let $\Delta_{i}$ be any neighborhood of $x$ that is carried by local coordinates onto a polydisk $\Delta^{m} \times \Delta^{n}, m>0$, with $\Delta_{i} \cap W=0 \times \Delta^{n}, \Delta^{m}$ and $\Delta^{n}$ being any polydisks in $\mathbf{C}^{m}$ and $\mathbf{C}^{n}$.

Let $g:[0,1] \times \Delta^{m} \times \Delta^{n} \rightarrow \Delta^{m} \times \Delta^{n}$ be given by $g(t, w, z)=(w, t z)$. Taking the associated homotopy operator we have $u=g_{0 *} u-d K_{g} u$ for any $u \in I_{r}\left(\Delta^{m}\right.$ $\left.\times \Delta^{n}\left\{0 \times \Delta^{n}\right\}\right)$ with $d u=0$. Then if we show that $K_{g} u \in I_{r+1}\left(\Delta^{m} \times \Delta^{n}\left\{0 \times \Delta^{n}\right\}\right)$ for any such $u$, then we will have shown that $u$ is homologous to the current $g_{0 *} u$, which is supported in $\Delta^{m} \times 0$. By the support property of $\S 7(\mathrm{a}), g_{0 *} u=i_{*} u_{0}, i$ the inclusion of $\Delta^{m}$ into $\Delta^{m} \times 0$, for a unique $u_{0} \in I_{r}\left(\Delta^{m}\right)$. By uniqueness $d u_{0}=0$.

Now if $r>2 m, u_{0}=0$ and thus $g_{0 *} u=0$. If $r=2 m$ then $u_{0} \in \mathscr{D}^{\prime 0}\left(\Delta^{m}\right)$, and $d u_{0}=0$ implies $u_{0}=[c]$, the current associated to a constant function, by Corollary $2.3(p=0)$. But $u_{0}$ is compactly supported so $c=0$ and $g_{0 *} u=0$. 
If $r<2 m$ by Definition 7.7 we have $0 \notin$ supp $u_{0}$ and thus $u_{0} \in I_{r}\left(\Delta^{m}-\{0\}\right)$. But $H_{r}\left(I .\left(\Delta^{m}-\{0\}\right)\right) \simeq H_{r}^{c}\left(\Delta^{m}-\{0\}, \mathbf{Z}\right) \simeq \mathbf{Z}$ if $r=0,2 m-1$ and $\simeq 0$ if $r \neq 0,2 m-1$ by the case $W=\varnothing$ proved at first. Thus if $r \neq 0,2 m-1$, $u_{0}=d v, v \in I_{r+1}\left(\Delta^{m}-\{0\}\right)$ and $g_{0 *} u=d i_{*} v$. If $r=0,2 m-1, u_{0}=d v+k v_{0}$, $k \in \mathbf{Z}$, where $v \in I_{r+1}\left(\Delta^{m}-\{0\}\right)$ and $v_{0}=[\{y\}], y \in \Delta^{m}-0$, or $v_{0}=\left[S_{\varepsilon}\right]$, $S_{\varepsilon}=\{w:|w|=\varepsilon\}$, respectively. Since $i_{*} v \in I_{r+1}\left(\Delta^{m} \times \Delta^{n}\left\{0 \times \Delta^{n}\right\}\right)$, everything is proved except for the case $r=2 m-1$, when $u$ is homologous to $i_{*}\left[S_{\varepsilon}\right]$. But $i_{*}\left[S_{\varepsilon}\right]=-d i_{*}\left[B_{\varepsilon}\right]$, where $B_{\varepsilon}=\left\{w \in \Delta^{m}:|w| \leq \varepsilon\right\}$. Since, by Proposition 7.8, $i_{*}\left[B_{\varepsilon}\right] \in I_{2 m}\left(\Delta^{m} \times \Delta^{n}\left\{0 \times \Delta^{n}\right\}\right)$, we are done after we prove that $K_{g} u \in$ $I_{r+1}\left(\Delta^{m} \times \Delta^{n}\left\{0 \times \Delta^{n}\right\}\right)$.

We prove that if $v \in \mathscr{D}_{r}^{\prime}\left(\Delta^{m} \times \Delta^{n}\right)$ has intersection type along $0 \times \Delta^{n}$, then so does $K_{g} v$ and furthermore $K_{g}\left(v \cdot\left(0 \times \Delta^{n}\right)\right)=\left(K_{g} v\right) \cdot\left(0 \times \Delta^{n}\right)$. This clearly suffices since under the hypotheses of this lemma the current on the left-hand side is an integral current.

Let $g^{\prime}:[0,1] \times \hat{\Delta}^{m} \times \Delta^{n} \rightarrow \hat{\Delta}^{m} \times \Delta^{n}$ be given by $g^{\prime}(t, x, z)=(x, t z)$. As usual o: $\hat{\Delta}^{m} \times \Delta^{n} \rightarrow \Delta^{m} \times \Delta^{n}$ and $\sigma^{-1}\left(0 \times \Delta^{n}\right)=E$.

Given $v$ it is clear that $\left(K_{g} v\right)_{\sigma}=K_{g^{\prime}} v_{\sigma}=g_{*}^{\prime}\left([0,1] \times v_{\sigma}\right)$ since the latter is a current of order zero which agrees with the former on the complement of $E$ and for both of which $E$ is a set of measure zero. By the same reasoning $\left(K_{g^{\prime}} v\right)_{\sigma} \wedge \mu$ $=K_{g^{\prime}}\left(v_{\sigma} \wedge g^{*} \mu\right)$ for any $\mu \in A^{s}\left(\hat{\Delta}^{m} \times \Delta^{n}\{E\}\right)$. Notice that $g^{*} \mu$ is still of residue type if $\mu$ is.

Choose any $\mu$ such that $g^{*} \mu=\mu$ and Res $\mu=[E]$; for example $\mu$ $=(2 \pi i)^{-1} \sigma^{*} d^{\prime} \log \Sigma\left|w_{i}\right|^{2}$. Then, by Definition 7.6, $\left(K_{g^{\prime}} v_{0}\right) \cdot E$ $=(-1)^{r+1} \operatorname{res}\left(\left(K_{g^{\prime}} v_{0}\right) \wedge \mu\right)=(-1)^{r+1} K_{g^{\prime}}\left(\operatorname{res}\left(v_{\sigma} \wedge \mu\right)\right)=K_{g^{\prime}}\left(v_{0} \cdot E\right)$. This says that $\left(K_{g} v\right)_{\sigma} \cdot E$ is a product of the fiber $\mathrm{P}^{m-1}$ with $K_{g}\left(v \cdot\left(0 \times \Delta^{n}\right)\right)$ since $v_{\sigma} \cdot E$ is such a product. Thus, by Proposition 7.9, $K_{g} v$ is of intersection type and $\left(K_{g} v\right) \cdot\left(0 \times \Delta^{n}\right)=K_{g}\left(v \cdot\left(0 \times \Delta^{n}\right)\right)$.

Remark. Because of the application to [10] we have dealt only with Zhomology, but it is clear that the strong intersection currents, the currents $u$ such that $u$ and $d u$ are intersection type, give a homology (or cohomology) theory that agrees with the usual theory with coefficients in $\mathbf{C}$ and that pairs naturally with $\Phi^{\prime \prime}(V\{W\})$. One could also do Dolbeault theory along the lines of $\$ 3$.

(e) An intersection formula. All the essentials for showing (7.1) have now been established. In this section we will spell out what we have proved and how to complete the demonstration.

By Definition 7.6 any current $v$ of degree $s$ which has intersection type along $W$ satisfies

$$
(-1)^{s} i_{*}(\nu \cdot W \wedge \operatorname{res} \eta)=\operatorname{Res}(\nu \wedge \eta)=d(\nu \wedge \eta)-\left(\left.(d(\nu \wedge \eta))\right|_{\nu-W}\right)^{\sim}
$$

for any $\eta \in A^{r}(V\{W\})$. But since $v$ and $d v$ are current transversal,

$$
\left(\left.(d(v \wedge \eta))\right|_{v-W}\right)^{\sim}=d v \wedge \eta+(-1)^{s} v \wedge d \eta \text { for } v \in \Phi^{\prime s}(V) .
$$


Lemma 7.14. If $d v$ above is in $F^{p D^{\prime s}}(V)$ and $\eta \in F^{q} A^{r}(V\{W\})$, with $p+q$ $>\operatorname{dim} V$, then $i_{*}(v \cdot W \wedge$ res $\eta)=(-1)^{s} d(v \wedge \eta)-v \wedge d \eta$.

Proof. Since $d v \wedge \eta=0$, immediate from discussion above.

Theorem 7.15. Let $[X] \in \mathscr{D}_{2 k}^{\prime}(V)$ be the current defined by the complex subvariety $X \subset V$ with (positive and negative) integer multiplicities; and suppose that $[X]$ is homologous to zero in $H_{2 k}^{\Phi}(V, Z) \simeq H_{2 k}\left(I^{\Phi}(V)\right)$. Then if $[X] \cdot W$ is defined for a submanifold $W \subset V$ of codimension $d$, there is an integral current $c$ $\in I_{2 k+1}^{\Phi}(V\{W\})$ such that $d c=[X]$ and $d(c \cdot W)=[X] \cdot W$. If $\varphi$ is any closed form in $F^{k-d+1} A_{c}^{2 k-2 d+1}(W)$, then for any $\psi \in F^{k+1} A_{c}^{2 k+1}(V)$ which equals $i_{*}[\varphi]$ $+d v$ for some $v \in F^{k+1} \mathcal{G}^{\prime 2 k}(V)$,

$$
c(\psi)=c \cdot W(\varphi) .
$$

Remark. This means that $i_{*}[\varphi]$ and $\psi$ are cohomologous in $H_{c}^{k}\left(V, \hat{\Omega}^{k+1}\right)$; by Theorem 2.13 we can choose $v=[\eta], \eta \in F^{k+1} A_{c}^{2 k}(V\{W\})$. Then $\operatorname{res}[\eta]=\varphi$ and $-d \eta=\psi$.

Proof. We can choose such a $c$ by Theorem 7.11. The equality is true by Lemma 7.14 having chosen $\eta$ with $i_{*}[\varphi]-\psi=d[\eta]$ by the remark above.

8. Residues in cohomology. If $W \subset V$ is a complex submanifold of codimension $d$, there is a long exact sequence of local cohomology:

$$
\cdots \rightarrow H_{W}^{r}(V, \mathrm{C}) \rightarrow H^{r}(V, \mathrm{C}) \rightarrow H^{r}(V-W, \mathrm{C}) \rightarrow \cdots
$$

where there is the natural Thom isomorphism $H_{W}^{r}(V, \mathrm{C})=H^{r-2 d}(W, \mathrm{C})$ since $W$ is nonsingular.

We would like to express this in terms of residue forms and currents. Take the resolutions:

(a) $0 \rightarrow \mathbf{C}_{W} \rightarrow \mathfrak{A}_{W}^{0} \stackrel{d}{\rightarrow} \mathfrak{A}_{W}^{1} \stackrel{d}{\rightarrow} \cdots$ by $C^{\infty}$ forms on $W$ given by (2.4)(i).

(b) $0 \rightarrow \mathbf{C}_{V} \rightarrow K_{V\{W\}}^{0} \stackrel{d}{\rightarrow} K_{V\{W\}}^{1} \stackrel{d}{\rightarrow} \cdots$ by residue currents on $V$ given by (2.11a).

Also the complex of sheaves

(c) $\mathfrak{U}_{V\{W\}}^{0} \stackrel{D}{\longrightarrow} \mathfrak{X}_{V\{W\}}^{\prime} \rightarrow \cdots$ where $\mathfrak{U}_{V(W\}}^{r}$ is the sheaf on $V$ of germs of forms in $A_{V(W)}^{r}$. We stress that this complex is not exact for the operator $D \eta=d \eta$ (not $d[\eta]$ as in (b)). The cohomology sheaf of this complex is $\mathbf{C}_{V}$ in dimension $\mathbf{0}$ and $\mathrm{C}_{W}$ in dimension $2 d-1$. By a spectral sequence argument, since this agrees with the local cohomology sheaves $\mathcal{T}_{V-W}^{i}(V, \mathrm{C})$ on $V$ we have:

Proposition 8.2.

$$
H_{V-W}^{r}(V, \mathrm{C}) \simeq H^{r}(V-W, \mathrm{C}) \simeq \frac{\operatorname{Ker}\left(D: A^{r}(V\{W\}) \rightarrow A^{r+1}(V\{W\})\right)}{D\left(A^{r-1}(V\{W\})\right)}
$$

Proof. As indicated above, use spectral sequences or use Theorem 6.5 of [25]. 
We write this latter de Rham group as $H^{r}\left(A^{\prime}(V\{W\})\right)$ and the groups for (a) and (b) as $H^{r}\left(A^{\prime}(W)\right)$ and $H^{r}\left(\Phi^{\prime \prime}(V\{W\})\right)$. Then we have a long exact sequence:

$$
\rightarrow H^{r-2 d}\left(A^{\cdot}(W)\right) \stackrel{i *}{\rightarrow} H^{r}\left(\mathscr{D}^{\prime \prime}(V\{W\})\right) \stackrel{\rho}{\rightarrow} H^{r}\left(A^{\prime}(V\{W\})\right) \rightarrow \cdots
$$

which corresponds to the sequence (8.1) under the isomorphisms induced by (a), (b), (c).

The maps are induced by the maps of complexes:

(i) $i_{*}: A^{r-2 d}(W) \rightarrow \Phi^{\prime r}(V\{W\})$ given by $\varphi \rightarrow i_{*}[\varphi], i: W \rightarrow V$ being the inclusion.

(ii) $\rho: \mathscr{D}^{\prime r}(V\{W\}) \rightarrow A^{r}(V\{W\})$ is the restriction to $V-W$ followed by simple extension.

(iii) res: $A^{r}(V\{W\}) \rightarrow A^{r-2 d+1}(W)$ takes $\eta \rightarrow \operatorname{res}[\eta]$.

The maps (i) and (ii) induce the corresponding maps in (8.3) and the map of (iii) induces the coboundary map:

$$
H^{r}(A \cdot(V\{W\})) \stackrel{\text { res }}{\longrightarrow} H^{r-2 d+1}\left(A^{\cdot}(W)\right)
$$

corresponding to $H^{r}(V-W, \mathrm{C}) \stackrel{\&}{\rightarrow} H_{W}^{r+1}(V, \mathrm{C})$.

There is also a sequence dual to (8.1).

$$
\cdots \leftarrow H_{c}^{2 n-r}(W, \mathrm{C}) \stackrel{i^{*}}{\leftarrow} H_{c}^{2 n-r}(V, \mathrm{C}) \stackrel{j_{*}}{\longleftarrow} H_{c}^{2 n-r}(V-W, \mathrm{C}) \stackrel{\&}{\longleftarrow} \cdots
$$

If these spaces are represented by the de Rham groups of smooth forms, the pairing of forms in $A_{c}^{2 n-r}(V)$ and the currents in $\mathscr{D}^{\prime r}(V\{W\})$ induces the pairing between (8.4) and (8.1).

However, if we are interested in writing the dual sequence to (8.1) as homology:

$$
\cdots \leftarrow H_{r-2 d}^{c}(W) \stackrel{i^{*}}{\leftarrow} H_{r}^{c}(V) \stackrel{j_{*}^{*}}{\leftarrow} H_{r}^{c^{\prime}}(V-W) \leftarrow \cdots
$$

where $c^{\prime}$ means supports $K \subset V-W$ such that $\bar{K} \subset V$ is compact.

We can get a pairing with the currents in $\mathscr{D}^{\prime r}(V\{W\})$ if we represent homology by the strong intersection currents mentioned at the end of $\$ 7$. For $\mathbf{Z}$ homology we get a pairing using $I_{r}(V\{W\})$. The map $i^{*}$ in $(8.5)$ is given by intersection with $W ; j_{*}$ is the inclusion of the currents with zero intersection with $W$. The boundary map is given by taking the local product of the current with the fiber of the normal sphere bundle of $W$ (the dual to integrating forms over the fiber) and imbedding this in $V-W$.

This construction is well known (cf. [26]) but it seemed worthwhile to point out that it fits very naturally and rigorously into the theory developed in this paper.

\section{BIBLIOGRAPHY}

1. P. Baum and R. Bott, On the zeroes of meromorphic vector-fields, Essays on Topology and Related Topics (Mémoires dédiés à Georges de Rham), Springer, New York, 1970, pp. 29-47. MR 41 \#6248.

2. T. Bloom and M. Herrera, De Rham cohomology of an analytic space, Invent. Math. 7 (1969), 275-296. MR 40 \# 1601. 
3. A. Borel and A. Haefliger, La classe d'homologie fondamentale d'un espace analytique, Bull. Soc. Math. France 89 (1961), 461-513. MR 26 \#6990.

4. G. Bredon, Sheaf theory, McGraw-Hill, New York, 1967. MR 36 \#4552.

5. Michael J. Cowen, Hermitian vector bundles and value distribution for Schubert cycles, Trans. Amer. Math. Soc. 180 (1973), 189-228.

6. P. Dolbeault, Formes différentielles et cohomologie sur une variété analytique complexe. I, Ann. of Math. (2) 64 (1956), 83-130. MR 18, 670.

7. R. N. Draper, Intersection theory in analytic geometry, Math. Ann. 180 (1969), 175-204. MR 40 \#403.

8. H. Federer, Geometric measure theory, Die Grundlehren der math. Wissenschaften, Band 153, Springer-Verlag, New York, 1969. MR 41 \# 1976.

9. R. Godement, Topologie algébrique et théorie des faisceaux, Actualités Sci. Indust., no. 1252, Hermann, Paris, 1958. MR 21 \# 1583.

10. P. Griffiths, Some results on algebraic cycles on algebraic manifolds, Algebraic Geometry (Internat. Colloq., Tata Inst. Fund. Res., Bombay, 1968), Oxford Univ. Press, London, 1969, pp. 93-191. MR 41 \# 1746.

11. R. Hartshorne, Local cohomology, A Seminar given by A. Grothendieck, Harvard University, Fall, 1961, Lecture Notes in Math., no. 41, Springer-Verlag, Berlin and New York, 1967. MR 37 \#219.

12. R. C. Gunning and H. Rossi, Analytic functions of several complex variables, Prentice-Hall Series in Modern Analysis, Prentice-Hall, Englewood Cliffs, N.J., 1965. MR 31 \#4927.

13. M. Herrera, Résidus multiples sur les espaces complexes, 1972 (preprint).

14. M. Herrera and D. Lieberman, Residues and principal values on complex spaces, Math. Ann. 194 (1971), 259-294.

15. H. Hironaka, Resolution of singularities of an algebraic variety over a field of characteristic zero. I, II, Ann. of Math. (2) 79 (1964), 109-326. MR 33 \#7333.

16. F. Hirzebruch, Neue topologische Methoden in der algebraischen Geometrie, Ergebnisse der Mathematik und ihrer Grenzgebiete, Heft 9, Springer-Verlag, Berlin, 1956; English transl., Die Grundlehren der math. Wissenschaften, Band 131, Springer-Verlag, New York, 1966. MR 18, 509; 34 \#2573.

17. L. Kaup, Eine Künnethformel für Fréchetgarben, Math. Z. 97 (1967), 158-168. MR 35 \#6868.

18. J. King, A residue formula for complex subvarieties, Proc. Carolina Conf. on Holomorphic Mappings and Minimal Surfaces (Chapel Hill, N.C., 1970), Department of Mathematics, University of North Carolina, Chapel Hill, N.C., 1970, pp. 43-56. MR 42 \#7942.

19._, The currents defined by analytic varieties, Acta Math. 127 (1971), 185-220.

20. The Abel-Jacobi map for families of Kahler manifolds (to appear).

21. S. Kobayashi and K. Nomizu, Foundations of differential geometry. Vol. II, Wiley, New York, 1969.

22. P. Lelong, Fonctionnelles analytiques et fonctions entières, Université de Montréal, 1967.

23. - Fonctions plurisousharmoniques et formes différentielles positives, Gordon and Breach, New York; distributed by Dunod, Paris, 1969. MR 39 \#4436.

24.__ Séminaire Pierre Lelong, 1970, Springer-Verlag, New York, 1971.

25. R. Narasimhan, Compact analytical varieties, Enseignement Math. (2) 14 (1968), 75-98. MR 39 \#3530.

26. F. Norguet, Introduction d la théorie cohomologique des résidus, Séminaire Pierre Lelong, 1970, Springer-Verlag, New York, 1971.

27. J.-B. Poly, Sur un théorème de J. Leray en théorie des résidus, C. R. Acad. Sci. Paris Sér. A-B 274 (1972), Al71-A174. MR 44 \#6994.

28. G. de Rham, Variétés différentiables. Formes, courants, formes harmoniques, Actualités Sci. Indust., no. 1222, Hermann. Paris, 1955. MR 16, 957. 
29. L. Schwartz, Thérie des distributions, Nouvelle Edition, entièrement corrigete refondue et augmenté, Hermann, Paris, 1966.

30. W. Stoll, Value distribution of holomorphic maps into compact complex manifolds, Lecture Notes in Math., vol. 135, Springer-Verlag, New York, 1970. MR 42 \#2040.

31. G. Stolzenberg, Volumes, limits, and extensions of analytic varieties, Lecture Notes in Math., no. 19, Springer-Verlag, New York, 1966. MR 34 \#6156.

School of Mathematics, Institute for Advanced Study, Prnnceton, New Jersey 08540

Current address: Department of Mathematics, Room 2-179, Massachusetts Institute of Technology, Cambridge, Massachusetts 02139 